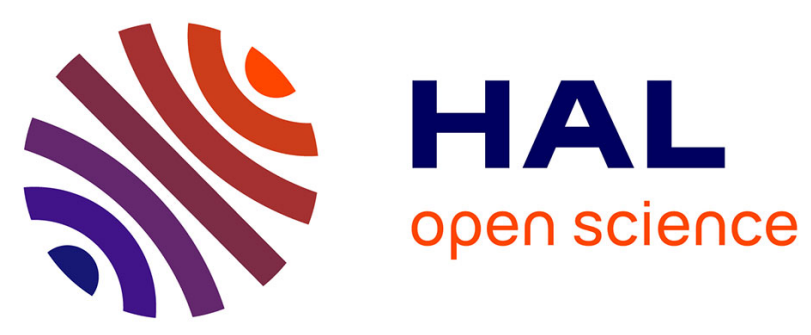

\title{
Evolution of nuclear shapes in odd-mass yttrium and niobium isotopes from lifetime measurements following fission reactions
}

T.W. Hagen, A. Görgen, W. Korten, L. Grente, M. Salsac, F. Fanny Farget, I. Ragnarsson, T. Braunroth, B. Bruyneel, I. Celikovic, et al.

\section{To cite this version:}

T.W. Hagen, A. Görgen, W. Korten, L. Grente, M. Salsac, et al.. Evolution of nuclear shapes in oddmass yttrium and niobium isotopes from lifetime measurements following fission reactions. Physical Review C, 2017, 95, pp.34302. 10.1103/PhysRevC.95.034302 . cea-01511760

\section{HAL Id: cea-01511760 https://hal-cea.archives-ouvertes.fr/cea-01511760}

Submitted on 21 Apr 2017

HAL is a multi-disciplinary open access archive for the deposit and dissemination of scientific research documents, whether they are published or not. The documents may come from teaching and research institutions in France or abroad, or from public or private research centers.
L'archive ouverte pluridisciplinaire HAL, est destinée au dépôt et à la diffusion de documents scientifiques de niveau recherche, publiés ou non, émanant des établissements d'enseignement et de recherche français ou étrangers, des laboratoires publics ou privés. 


\title{
Evolution of nuclear shapes in odd-mass yttrium and niobium isotopes from lifetime measurements following fission reactions
}

\author{
T. W. Hagen, ${ }^{1}$ A. Görgen,,${ }^{1}{ }^{*}$ W. Korten, ${ }^{2}$ L. Grente, ${ }^{2}$ M.-D. Salsac, ${ }^{2}$ F. Farget,${ }^{3}$ I. Ragnarsson, ${ }^{4}$ T. Braunroth,${ }^{5}$ B. Bruyneel, ${ }^{2}$ \\ I. Celikovic, ${ }^{3,6}$ E. Clément, ${ }^{3}$ G. de France, ${ }^{3}$ O. Delaune, ${ }^{3}$ A. Dewald, ${ }^{5}$ A. Dijon, ${ }^{3}$ M. Hackstein, ${ }^{5}$ B. Jacquot, ${ }^{3}$ J. Litzinger, ${ }^{5}$ \\ J. Ljungvall, ${ }^{7}$ C. Louchart, ${ }^{2}$ C. Michelagnoli, ${ }^{8}$ D. R. Napoli, ${ }^{8}$ F. Recchia, $,{ }^{9}, 10$ W. Rother, ${ }^{5}$ E. Sahin, ${ }^{1,8}$ S. Siem, ${ }^{1}$ \\ B. Sulignano, ${ }^{2}$ Ch. Theisen, ${ }^{2}$ and J. J. Valiente-Dobon ${ }^{8}$ \\ ${ }^{1}$ Department of Physics, University of Oslo, Oslo, Norway \\ ${ }^{2}$ Irfu, CEA, Université Paris-Saclay, F-91191 Gif-sur-Yvette, France \\ ${ }^{3}$ Grand Accélérateur National d'Ions Lourds (GANIL), CEA/DRF - CNRS/IN2P3, Boulevard Henri Becquerel, 14076 Caen, France \\ ${ }^{4}$ Division of Mathematical Physics, LTH, Lund University, Lund, Sweden \\ ${ }^{5}$ Institut für Kernphysik, Universität zu Köln, Köln, Germany \\ ${ }^{6}$ Institute of Nuclear Sciences "Vinca”, University of Belgrade, Belgrade, Serbia \\ ${ }^{7}$ CSNSM, IN2P3, Orsay, France \\ ${ }^{8}$ Laboratori Nazionali di Legnaro, INFN, Legnaro, Italy \\ ${ }^{9}$ Dipartimento di Fisica e Astronomia, Università degli Studi di Padova, Padova, Italy \\ ${ }^{10}$ INFN Sezione di Padova, Padova, Italy
}

(Received 19 November 2016; published 3 March 2017)

\begin{abstract}
Lifetimes of excited states in ${ }^{99} \mathrm{Y},{ }^{101} \mathrm{Y},{ }^{101} \mathrm{Nb},{ }^{103} \mathrm{Nb}$, and ${ }^{105} \mathrm{Nb}$ were measured in an experiment using the recoil distance Doppler shift method at GANIL (Grand Accélérateur National d'Ions Lourds). The neutron-rich nuclei were produced in fission reactions between a ${ }^{238} \mathrm{U}$ beam and a ${ }^{9} \mathrm{Be}$ target. Prompt $\gamma$ rays were measured with the EXOGAM array and correlated with fission fragments that were identified in mass and atomic number with the VAMOS ++ spectrometer. The measured lifetimes, together with branching ratios, provide $B(M 1)$ and $B(E 2)$ values for the strongly coupled rotational bands built on the $[422] 5 / 2^{+}$ground state in the $\mathrm{Y}$ and $\mathrm{Nb}$ nuclei with neutron number $N \geqslant 60$. The comparison of the experimental results with triaxial particle-rotor calculations provides information about the evolution of the nuclear shape in this mass region.
\end{abstract}

DOI: 10.1103/PhysRevC.95.034302

\section{INTRODUCTION}

Interesting physics is often to be found when sudden deviations from a general trend occur. The properties of atomic nuclei with partially filled proton and neutron shells tend to change smoothly as a function of proton number $Z$ and neutron number $N$. In nuclei with $Z \approx 40$ and $N \approx 60$, however, a sudden onset of deformation is observed. For the even-even nuclei of $\operatorname{Sr}(Z=38)$ and $\operatorname{Zr}(Z=40)$, the sudden drop in the excitation energy of the first $2^{+}$state and the corresponding steep increase of the $B\left(E 2 ; 0_{1}^{+} \rightarrow 2_{1}^{+}\right)$values [1] between $N=58$ and $N=60$ are clear indications of the dramatic onset of deformation in these nuclei. Further evidence comes from the nonlinear increase of charge radii observed for the chains of $\mathrm{Rb}, \mathrm{Sr}, \mathrm{Y}, \mathrm{Zr}$, and $\mathrm{Nb}$ isotopes [2]. The lower boundary for the region of rapid shape change is formed by the chain of $\mathrm{Kr}$ isotopes, for which the charge radii [3] and $2_{1}^{+}$energies and $B(E 2)$ values [4] evolve smoothly.

The onset of deformation for the $\mathrm{Zr}$ isotopes at $N=60$ was first explained by Sheline et al. as the inversion of spherical and strongly deformed intruder configurations with shape coexistence in the transitional region [5]. Evidence for shape coexistence in the $\mathrm{Sr}$ and $\mathrm{Zr}$ nuclei with $N=58$ and $N=60$ comes from the observation of low-lying $0_{2}^{+}$states and strong

*andreas.gorgen@fys.uio.no electric monopole $(E 0)$ transitions [6-9]. The measurement of spectroscopic quadrupole moments for the $2_{1}^{+}$and $2_{2}^{+}$ states in ${ }^{96} \mathrm{Sr}$ and ${ }^{98} \mathrm{Sr}$ finally provided direct evidence for shape coexistence and the inversion of the ground-state shape from near spherical to strongly deformed at $N=60[10,11]$. Large shell gaps at quadrupole deformation $\beta \approx 0.4$ for proton numbers $Z=38$ and 40 and for neutron numbers $N=60$ and 62 reinforce the deformation [12]. Particle-hole excitations across the deformed shell gaps facilitate the occurrence of shape coexistence.

The sudden onset of deformation around $Z=40$ and $N=$ 60 has been explained by the depletion of protons from the orbitals of the $N=3$ oscillator shell and the filling of $\pi g_{9 / 2}$ orbitals combined with the occupation of the $v h_{11 / 2}$ neutron orbitals and depletion of neutrons in the $\nu g_{9 / 2}$ orbitals [13]. Indeed, such occupation changes are supported by standard Nilsson diagrams [14], although the neutron diagram suggests that the strongly deformed bands around $N=60$ and 62 are formed with the $g_{9 / 2}$ neutron shell essentially fully occupied. In general, however, holes in up-sloping Nilsson orbitals can be equally or even more important than particles in downsloping orbitals to create strongly collective bands, as has been emphasized for high-spin bands $[15,16]$. For heavier elements with $Z \geqslant 42$ the transition from spherical to deformed shape at $N=60$ becomes again more gradual. With additional protons above the deformed shell gap the ground-state deformation is expected to become smaller and triaxial shapes are expected to become increasingly important $[17,18]$. 
Nuclei with proton number $Z \approx 40$ and mass $A \approx 100$ are produced in fission reactions, which makes them experimentally accessible despite their large neutron excess. The structure of nuclei in this region has been extensively studied in the past using high-fold $\gamma$-ray coincidence measurements with spontaneous fission sources placed in large germanium detector arrays [12]. This method relies on identifying $\gamma$-ray transitions in a given fission fragment by their coincidence relationships with known transitions in the same nucleus. Assigning $\gamma$-ray transitions to a specific nucleus can be challenging because of coincidence relationships with transitions in different isotopes of the binary fission fragment, depending on the number of neutrons emitted in the fission process. Nevertheless, extensive level schemes were obtained in this way, often with several rotational bands extending up to spin $I \sim 20$ in the strongly deformed nuclei of this mass region. Prompt $\gamma$-ray spectroscopy of fission fragments has also been performed following fission reactions induced by cold neutrons from a reactor [19]. Spectroscopy of very neutron-rich fission fragments was advanced recently by isotopic identification of the fission fragments produced in fusion-fission and transfer-induced fission reactions at energies around the Coulomb barrier using a large acceptance spectrometer coupled with a large $\gamma$-ray detector array at GANIL (Grand Accélérateur National d'Ions Lourds) [20,21].

Quantitative experimental information on observables related to the nuclear deformation is important to understand the evolution of nuclear structure in this region of rapid structural changes. Experimental measurements of electromagnetic transition strengths provide such information and serve as benchmarks to test theoretical model calculations and their predictive power. Experimentally, there are two complementary methods to obtain electromagnetic matrix elements, namely Coulomb excitation and lifetime measurements. Coulomb excitation experiments have only recently become feasible in this mass region with the development of fission fragment beams at radioactive ion beam facilities $[22,23]$. For ${ }^{96} \mathrm{Sr}$ and ${ }^{98} \mathrm{Sr}$, Coulomb excitation experiments have yielded reduced transition probabilities and spectroscopic quadruple moments for several low lying states, providing quantitative information about the shape transition and coexistence in these two nuclei $[10,11]$. Coulomb excitation experiments for ${ }^{97} \mathrm{Rb}$ and ${ }^{99} \mathrm{Rb}$ indicated that the sudden shape transition is also apparent in the chain of $\mathrm{Rb}$ isotopes and that their deformation is similar to the neighboring $\mathrm{Sr}$ and $\mathrm{Zr}$ isotopes with $N \geqslant 60$ [24].

Lifetime measurements for excited states in neutron rich fission fragments have been performed in the past by using mass-separated samples of fission fragments and $\beta$-delayed fast timing measurements using $\beta-\gamma-\gamma$ coincidences $[25,26]$. This method is suitable for relatively long lifetimes, typically above $\tau>50 \mathrm{ps}$. Lifetime measurements were also performed using spontaneous fission sources. In one variant, the so-called Doppler-profile method [27], the fission source is embedded in a stopper material and the lineshapes of the Doppler-broadened $\gamma$-ray transitions are compared to simulations of the stopping process, relating the lifetime of excited states with the stopping time of the fission fragments. This method is sensitive to short lifetimes of the order of $\tau \sim 1$ ps. In another variant, the fission source is mounted in a plunger device combined with an array of silicon detectors for the detection of fission fragments [28].

The rotational ground-state bands of ${ }^{99} \mathrm{Y}$ and ${ }^{101} \mathrm{Y}$ were first observed following the $\beta$-decay of mass-separated fragments from neutron-induced fission and were interpreted as strongly coupled bands based on the [422]5/2 $2^{+}$Nilsson orbital $[29,30]$. Studies of high-fold $\gamma$-ray coincidences have yielded extensive level schemes including three-quasiparticle excitations and negative-parity bands [31]. The ground-state bands in ${ }^{99} \mathrm{Y}$ and ${ }^{101} \mathrm{Y}$ show very little signature splitting compared to the Tc and $\mathrm{Rh}$ isotopes with neutron numbers $N=60$ and $N=62$, which has been interpreted as an indication of axially symmetric shape of the $\mathrm{Y}$ isotopes [31]. The comparison with particle-rotor calculations supports axial symmetry for ${ }^{99} \mathrm{Y}$ and ${ }^{101} \mathrm{Y}$ and suggests a large quadrupole deformation of $\epsilon_{2} \approx 0.4$ [31]. The spectroscopic quadrupole moment and the magnetic moment of the ground state have been measured for the entire chain of $\mathrm{Y}$ isotopes using laser spectroscopy [32]. The results clearly show the sudden onset of deformation at $N=60$ and yielded electric quadrupole moments for ${ }^{99} \mathrm{Y}$ and ${ }^{101} \mathrm{Y}$ that correspond to large prolate deformation, whereas the magnetic moments are consistent with a proton in the [422]5/2+ Nilsson orbital [32]. The lifetime of the first excited $7 / 2^{+}$state in ${ }^{99} \mathrm{Y}$ was measured to be $\tau=68(9)$ ps [33]. However, without knowledge of the $E 2 / M 1$ mixing ratio $\delta$, no reliable $B(E 2)$ and $B(M 1)$ values can be obtained from this lifetime alone. To learn more about the underlying nuclear shape and its evolution with angular momentum, proton, and neutron number, it is essential to measure electromagnetic transition probabilities within the rotational bands. From the lifetimes of the higher-lying states and their $E 2 / M 1$ branching ratios it is possible to determine absolute $B(E 2)$ and $B(M 1)$ values, which can be compared to theoretical calculations.

The rotational ground-state bands in ${ }^{101} \mathrm{Nb}$ and ${ }^{103} \mathrm{Nb}$ were also first identified using $\beta$-delayed spectroscopy of mass-separated fragments following neutron-induced fission $[34,35]$. The rotational ground-state band of ${ }^{105} \mathrm{Nb}$ was first identified in the analysis of high-fold $\gamma-\gamma$ coincidence data from a spontaneous fission source and assigned to ${ }^{105} \mathrm{Nb}$ due to its similarity to the band in ${ }^{103} \mathrm{Nb}$, the systematics of fission yields, and cross-correlations with transitions in several $\mathrm{Cs}$ isotopes [36]. Also for the $\mathrm{Nb}$ isotopes under study the ground-state bands are interpreted based on the $[422] 5 / 2^{+}$ Nilsson orbital [31]. The level schemes were later extended and include negative-parity bands in ${ }^{101} \mathrm{Nb}$ [31] and candidates for one- and two-phonon $\gamma$-vibrational bands in ${ }^{103} \mathrm{Nb}$ [37] and ${ }^{105} \mathrm{Nb}$ [38]. The signature splitting in the ground-state bands is larger in the $\mathrm{Nb}$ isotopes than in $\mathrm{Y}$, suggesting the presence of a modest triaxial deformation. Comparisons with particle-rotor calculations suggest slightly smaller quadrupole deformation compared to the $\mathrm{Y}$ isotopes and also indicate the presence of $\gamma$ deformation in the $\mathrm{Nb}$ isotopes [31]. The spectroscopic quadrupole moment and the magnetic moment for the chain of odd-mass $\mathrm{Nb}$ isotopes were measured up to ${ }^{103} \mathrm{Nb}$ [39]. The results are consistent with a smaller deformation compared to $\mathrm{Y}$ and with the [422]5/2+ configuration for the ground state. Lifetimes were measured for several low-lying states in both the ground-state band and the [301]3/2- and [303]5/2- bands in ${ }^{101} \mathrm{Nb}$ using fast-timing techniques [40]. The results indicate 
a similarly large deformation for all three bands, although the uncertainties are large. Lifetimes have been measured also for several states in the negative-parity bands of ${ }^{103} \mathrm{Nb}$ using fast-timing techniques [41]. In the ground-state band of ${ }^{103} \mathrm{Nb}$ only the lifetime of the first excited $7 / 2^{+}$state could be measured with this technique [41]. Without any E2 crossover transition, assumptions about the $E 2 / M 1$ mixing ratio of the $7 / 2^{+} \rightarrow 5 / 2^{+}$transition are necessary to obtain information about the deformation from this lifetime. Lifetimes of the $21 / 2^{+}$and $17 / 2^{+}$states in the ground-state band of ${ }^{103} \mathrm{Nb}$ were measured using the Doppler profile method [42], but measurements at intermediate spins are lacking. No lifetimes have been measured so far for ${ }^{105} \mathrm{Nb}$.

Here we report on a lifetime measurement for excited states in the ground-state bands of ${ }^{99,101} \mathrm{Y}$ and ${ }^{101,103,105} \mathrm{Nb}$ using a novel technique combining the recoil-distance Doppler shift (RDDS) method $[43,44]$ with fission reactions in inverse kinematics and the unique identification of the fission fragments in a magnetic spectrometer. The inverse reaction kinematics produces fission fragments at large recoil velocities that can be used to measure lifetimes down to a few picoseconds using the RDDS method. The objective of the presented experiment was to determine electromagnetic transition probabilities for a wide range of nuclei in this area of the nuclear chart where rapid shape transitions, shape coexistence, and triaxiality occur. The experimental setup and the methods used are described in detail in Sec. II. The lifetime analysis and the results are presented in Sec. III. The results are discussed and compared to triaxial particle-rotor calculations in Sec. IV, followed by a summary and conclusions in Sec. V.

\section{EXPERIMENTAL DETAILS AND METHODS}

In an experiment performed at GANIL the magnetic spectrometer VAMOS $++[45]$ was coupled to the EXOGAM array of segmented Ge clover detectors [46]. A beam of ${ }^{238} \mathrm{U}$ ions at an energy of $6.2 \mathrm{~A} \mathrm{MeV}$ and intensity of $0.3 \mathrm{p} \mathrm{nA}$ was incident on a ${ }^{9}$ Be target of $2.3 \mathrm{mg} / \mathrm{cm}^{2}$ thickness. Fission fragments were mostly produced by fusion-fission reactions, producing the compound nucleus ${ }^{247} \mathrm{Cm}$ at an excitation energy of approximately $45 \mathrm{MeV}$ and resulting in a near-symmetric fragment distribution. To a lesser extent fragments were also produced in transfer-fission reactions [47]. The time scale of the fission reactions is short compared to the electromagnetic decay of the fission products. The fission fragments are strongly focused in the forward direction due to the inverse kinematics of the reaction, resulting in large recoil velocities and a high yield into the VAMOS++ spectrometer, which was placed at an angle of $20^{\circ}$ with respect to the beam axis.

Lifetimes of short-lived excited states in the fission fragments were measured using the RDDS technique with a compact plunger device [44] that was built for use with the VAMOS ++ spectrometer. In the plunger device the target and a degrader foil are stretched onto conical frames to obtain flat and even surfaces which can be moved relative to one another. The distance between the two foils is kept constant by an active feedback mechanism based on the measurement of the capacitance between the two foils. For the degrader a $4.9 \mathrm{mg} / \mathrm{cm}^{2}$ thick $\mathrm{Mg}$ foil was used. Data were taken for seven distances $(37,63,112,212,412,754$, and $1554 \mu \mathrm{m})$ for approximately 24 hours per distance. The degrader thickness was chosen as a compromise between larger differences in the Doppler shift of $\gamma$ rays emitted before and after passing the degrader and less energy and angular straggling that reduces the mass resolution in VAMOS ++ and the energy resolution in EXOGAM. A compromise is also made with regards to the choice of target-degrader distances. The aim to measure a wide range of lifetimes in a multitude of nuclides reduces the number of sensitive data points for a given $\gamma$-ray transition to typically 2 or 3 .

\section{A. The VAMOS ++ spectrometer and particle identification}

The large-acceptance spectrometer VAMOS ++ [45] comprises two quadrupole magnets and one dipole magnet. The focal-plane setup consisted of a multiwire parallel plate avalanche counter (MWPPAC), two drift chambers, a segmented ionization chamber, and a wall of silicon detectors [48]. The focal plane detectors cover an area of $1000 \times 150 \mathrm{~mm}^{2}$. Another MWPPAC was mounted at the entrance of the spectrometer. Ions with mass $m$, velocity $v$, and charge $q$ are separated according to their magnetic rigidity,

$$
B \rho=\gamma \frac{m v}{q},
$$

where $\gamma$ is the Lorentz factor. The magnetic rigidity was set to $B \rho=1.17 \mathrm{Tm}$. The settings of $B \rho$ and the angle of VAMOS ++ were optimized to select the desired fission fragments while suppressing the amount of scattered particles from the degrader reaching the focal plane. The amount of scattered $\mathrm{Mg}$ ions from the degrader at the focal plane was further reduced by placing a $100 \mu \mathrm{g} / \mathrm{cm}^{2}$ thick gold foil on a $0.9 \mu \mathrm{m}$ thick mylar backing at the entrance of $\mathrm{VAMOS}++$, which resulted in a lower average charge state and hence larger magnetic rigidity of the $\mathrm{Mg}$ ions.

Fission fragments that reached the focal plane were uniquely identified in mass, charge, and atomic number on an event-by-event basis. The time of flight through the spectrometer is measured between the two MWPPACs at the entrance and in the focal plane of VAMOS ++ . Signals from the focal-plane MWPPAC were used to trigger the data acquisition. The horizontal and vertical position and the direction of the ions were measured in the two drift chambers. With this information the trajectories of the ions through the spectrometer could be traced and their velocity and direction at the target position can be determined [45,49]. The energy loss of the ions was measured in the ionization chamber, which is segmented into three consecutive rows with five horizontal segments each. Each row of the ionization chamber has an active volume of $1000 \times 150 \times 120 \mathrm{~mm}^{3}$ and was filled with $\mathrm{CF}_{4}$ gas at 30 mbar pressure. The residual energy of the ions was measured in a wall of 40 silicon detectors of dimension $50 \times 80 \mathrm{~mm}^{2}$ and $500 \mu \mathrm{m}$ thickness. To calibrate the focal plane detectors the spectrometer was rotated to $45^{\circ}$ with respect to the beam axis and the ${ }^{9} \mathrm{Be}$ target and the degrader foil were replaced by a $1.3 \mathrm{mg} / \mathrm{cm}^{2}$ thick ${ }^{93} \mathrm{Nb}$ target. The elastically scattered ${ }^{93} \mathrm{Nb}$ ions were easily identified and used for calibration of the focal-plane detectors. 


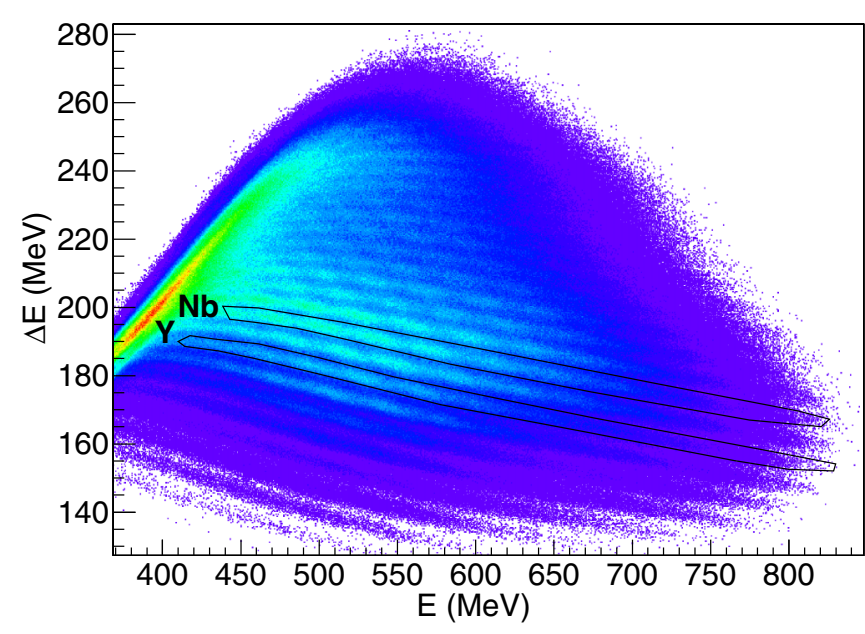

FIG. 1. Energy loss $\Delta E$ measured in the ionization chamber versus the total energy $E$ measured in the ionization chamber and the silicon wall. The cuts used for selecting $\mathrm{Y}$ and $\mathrm{Nb}$ ions are indicated.

The atomic number $Z$ of the fission fragments was determined from their characteristic energy loss in the ionization chamber and their total energy measured in the ionization chamber and the silicon wall. Figure 1 shows a plot of the energy loss $\Delta E$ versus the total energy $E$. Fission fragments with atomic numbers ranging from $Z=32$ to $Z=54$ could be identified. The distribution of fission fragments that reached the focal plane had a maximum for Mo $(Z=42)$. The mass number $A$ and the charge state $q$ of the ions are determined from the time of flight through the spectrometer and the magnetic rigidity obtained from the position of the ions in the focal plane. A plot of the mass versus the mass-to-charge ratio is presented in Fig. 2. Masses ranging from $A \approx 80$ to $A \approx 140$ could be identified in the experiment. In total it was possible to identify almost 400 different nuclides. Ions with five different charge states for a given nuclide typically

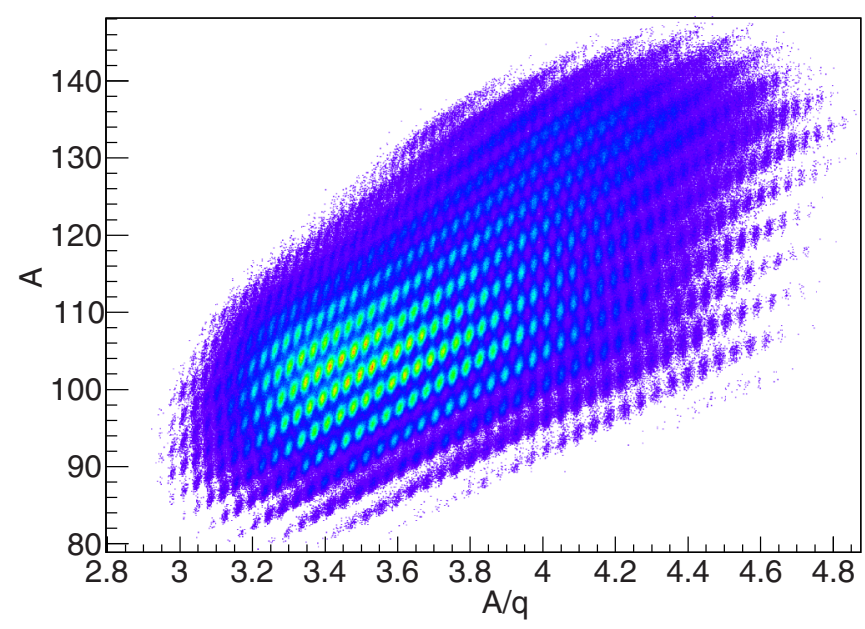

FIG. 2. Mass versus mass-to-charge ratio obtained from the magnetic rigidity of the ions and their time of flight through VAMOS ++ . The diagonal bands of loci correspond to a unique charge state.
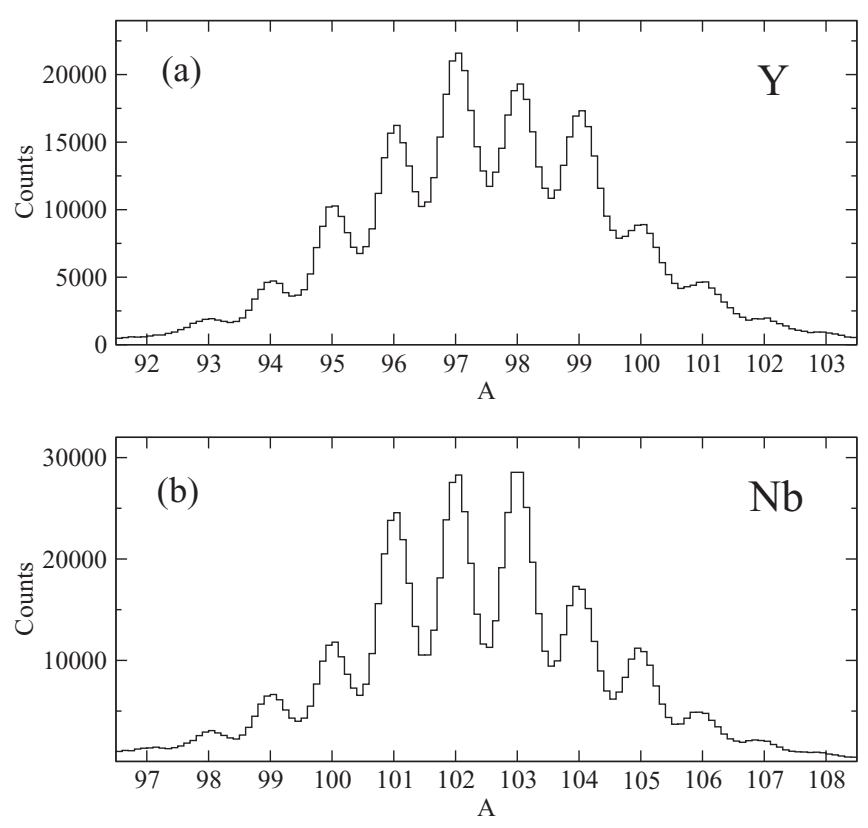

FIG. 3. Mass distributions of fission fragments identified as $\mathrm{Y}$ (a) and $\mathrm{Nb}(\mathrm{b})$.

reached the focal plane. The mass distributions for the $\mathrm{Y}$ and $\mathrm{Nb}$ isotopes of interest for the present study are shown in Fig. 3. The resolution in mass and atomic number achieved with the VAMOS ++ spectrometer allows selecting $\gamma$ rays originating from a given fission fragment.

\section{B. EXOGAM and the RDDS method}

The EXOGAM array of segmented Ge clover detectors [46] was placed around the target position. For the experiment the array consisted of 10 Compton-suppressed clover detectors at a distance of $147 \mathrm{~mm}$ from the target. Each clover detector comprises four Ge crystals, and the outer electrical contacts of each crystal are longitudinally segmented into four sectors, improving the angular resolution of the detectors. Three of the clover detectors were located at backwards angles of $135^{\circ}$ with respect to spectrometer axis and were hence sensitive to Doppler shifts. The remaining seven detectors were placed at $90^{\circ}$ and were used to identify $\gamma$-ray transitions in the fission fragments and to determine their relative intensities.

The measured energy of a $\gamma$ ray from a transition with transition energy $E_{0}$ is

$$
E_{\gamma}=E_{0} \frac{\sqrt{1-\beta^{2}}}{1-\beta \cos \theta},
$$

where $\theta$ is the angle between the direction of the fission fragment moving with velocity $\beta=v / c$ and the emitted $\gamma$ ray. The $\gamma$-ray spectra were Doppler corrected event by event by using the velocity vector of the fission fragments as measured in VAMOS ++ and the angle of the $\gamma$ rays obtained from the segments of the EXOGAM detectors. Because the degrader foil reduced the velocity of the fission fragments, the procedure is only correct for $\gamma$ rays emitted after the degrader. When $\gamma$ rays are emitted before the degrader foil at higher velocities, 


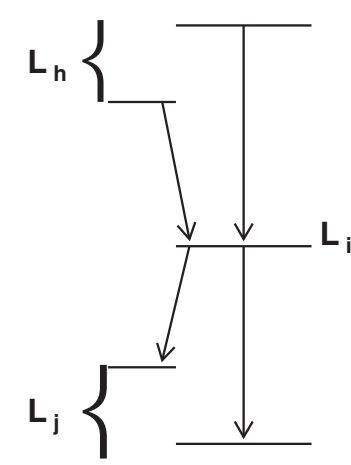

FIG. 4. Schematic representation of a typical decay scheme of a strongly coupled band where the state of interest $L_{i}$ is fed from the states $L_{h}$ and decays to the states $L_{j}$.

the Doppler corrected $\gamma$-ray energy is less than the transition energy $E_{0}$ when recorded in the detectors at backward angles. By comparing the relative intensities of the shifted (decay between target and degrader) and unshifted (decay behind the target) components of a transition at different target-degrader distances, the lifetime of excited states can be determined. In a $\gamma$-ray singles measurement the lifetimes of the feeding states have to be taken into account.

Figure 4 shows a schematic representation of a level scheme where $L_{i}$ is the state of interest which is fed from the higherlying states $L_{h}$ and decays to the states $L_{j}$ below. The situation shown in Fig. 4 is typical for strongly coupled bands as found in the $\mathrm{Y}$ and $\mathrm{Nb}$ isotopes under study. The so-called decay curve of a transition from the state $L_{i}$ to a specific state $L_{j}$ as a function of the target-degrader distance $x$ is given by

$$
Q_{i j}(x)=\frac{J_{i j}^{u}(x)}{J_{i j}^{u}(x)+J_{i j}^{s}(x)},
$$

where $J_{i j}^{s}(x)$ and $J_{i j}^{u}(x)$ are the intensities of the shifted and unshifted components, respectively, of the transition at the distance $x$. The corresponding decay curves $Q_{h i}(x)$ are defined in the same way for the feeding transitions. The lifetime $\tau_{i}$ of the state $L_{i}$ is found as [43]

$$
\tau_{i}=-\left[v_{0} \frac{d Q_{i j}(x)}{d x}\right]^{-1}\left[Q_{i j}(x)-b_{i j} \sum_{h} \alpha_{h i} Q_{h i}(x)\right],
$$

where $v_{0}$ is the velocity of the fission fragment before the degrader, $b_{i j}$ is the branching ratio for the decay transition under consideration, and $\alpha_{h i}$ is the relative strength of the feeding transitions, determined from the total intensities $J_{h i}$ and $J_{i j}$ and corrected for the relative detection efficiency.

The intensities of the shifted and unshifted components, $J^{s}(x)$ and $J^{u}(x)$, were determined by fitting the lineshape of the transition with two Gaussians. The centroid and the width of the shifted and unshifted peaks were determined using data for the longest and shortest distance, respectively, where most of the intensity is concentrated in one single component. The centroid and width was kept constant for all other distances where the transition strength is shared between the two components. Decay curves $Q_{i j}(x)$ were determined by the relative strength of the unshifted component as given in Eq. (3). The slope of the decay curve, $d Q_{i j}(x) / d x$, was obtained by fitting polynomial splines of second order and taking the derivative.

States in a strongly coupled rotational band can decay by a $\Delta I=1$ transition of mixed $M 1 / E 2$ multipolarity or by a $\Delta I=2 E 2$ transition. The decay curves for the two transitions depopulating the same state should be identical. Because the decay via mixed $M 1 / E 2$ transitions is dominating the rotational bands studied in this work, the decay curves for the $\Delta I=1$ transitions were used to extract the lifetimes, except in cases where another transition of similar energy made this impossible. To account for the feeding time, a weighted sum of the decay curves for the feeding transitions has to be subtracted as given in Eq. (4). The branching ratio $b_{i j}$ for the decay of the state of interest is independent of how the state was populated. In most cases the branching ratios were taken from the high-fold $\gamma-\gamma$ coincidence data of Luo et al. [31], where the energy resolution is better and the level of background lower compared to the present experiment. The uncertainties of the transition intensities are not explicitly given in Ref. [31]. As a conservative estimate, the relative intensity is assumed to have 5\% uncertainty for the strongest transitions which carry more than $10 \%$ of the $7 / 2^{+} \rightarrow 5 / 2^{+}$transition, and $10 \%$ for the weaker ones. Contrary to the branching ratio of the decay for the state of interest, the relative intensity of the feeding transitions $\alpha_{h i}$ is reaction dependent and consequently extracted from the present experiment using the detectors at $90^{\circ}$. In cases of weak feeding from $E 2$ transitions from a state $L_{h}$, the feeding correction was performed using the intensity of the $E 2$ transition and the decay curve of the corresponding $\Delta I=1$ transition depopulating the same state $L_{h}$.

The determination of lifetimes requires the knowledge of the initial velocity $v_{0}$ of the fission fragments before passing through the degrader. The detection of the fission fragments in VAMOS++ allows the reconstruction of the degraded velocity $v_{d}$ after the degrader foil. There are two possible ways to determine the initial velocity $v_{0}$ from the degraded velocity $v_{d}$ : The energy loss of the fission fragments in the degrader can be calculated using tabulated stopping powers of heavy ions in matter [50], or the velocity $v_{0}$ can be obtained from the experimental energy shift between the two components of a transition using Eq. (2). Because systematic uncertainties may be present in the method relying on tabulated stopping powers, we chose to use the energy difference between the two components to determine the velocity $v_{0}$. Due to the large momentum acceptance of VAMOS ++ , the velocity distribution of the fission fragments is rather broad (see Table I). A recent investigation demonstrated that the centroid

TABLE I. Centroids of the velocity distributions before and after the degrader, $v_{0}$ and $v_{d}$, respectively, and centroid of the inverse velocity distribution before the degrader, where $\beta_{0}=v_{0} / c$.

\begin{tabular}{llllll}
\hline \hline & \multicolumn{1}{c}{${ }^{99} \mathrm{Y}$} & \multicolumn{1}{c}{${ }^{101} \mathrm{Y}$} & \multicolumn{1}{c}{${ }^{101} \mathrm{Nb}$} & \multicolumn{1}{c}{${ }^{103} \mathrm{Nb}$} & ${ }^{105} \mathrm{Nb}$ \\
\hline$\left\langle v_{d}\right\rangle(\mu \mathrm{m} / \mathrm{ps})$ & 30.0 & 29.4 & 30.5 & 30.2 & 29.8 \\
$\left\langle v_{0}\right\rangle(\mu \mathrm{m} / \mathrm{ps})$ & $37.0(34)$ & $36.5(35)$ & $38.6(32)$ & $38.2(32)$ & $36.6(30)$ \\
$\left\langle 1 / \beta_{0}\right\rangle$ & $8.09(65)$ & $8.22(66)$ & $7.76(57)$ & $7.84(68)$ & $8.19(60)$ \\
\hline \hline
\end{tabular}




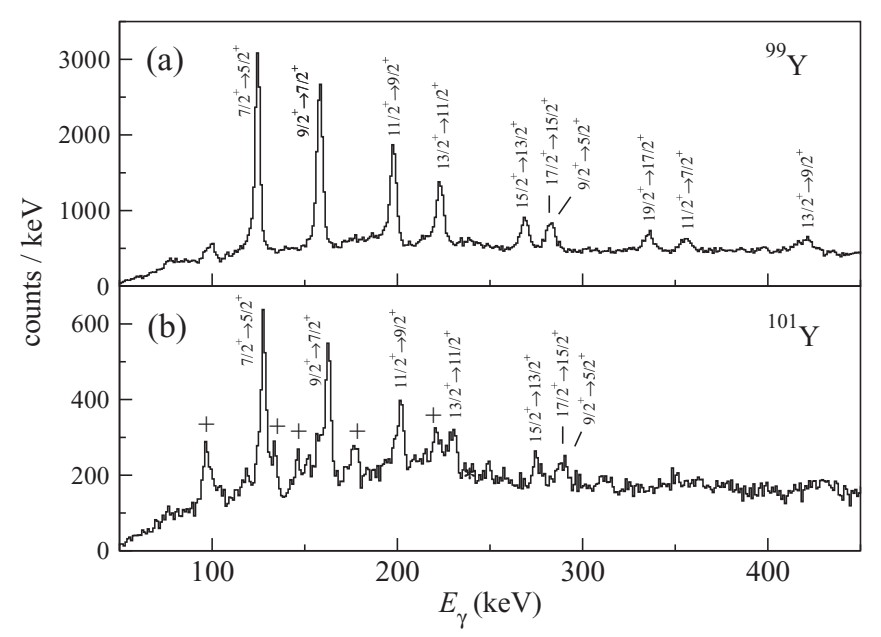

FIG. 5. Total $\gamma$-ray spectra in coincidence with fission fragments identified as ${ }^{99} \mathrm{Y}$ (a) and ${ }^{101} \mathrm{Y}$ (b), observed in the detectors mounted at $90^{\circ}$ with respect to the axis of VAMOS ++ , and summed over all seven target-degrader distances. The transitions marked by crosses originate from ${ }^{101} \mathrm{Zr}$, which is produced with much higher yield and contaminates the ${ }^{101} \mathrm{Y}$ spectrum.

of the inverse velocity distribution $\left\langle 1 / v_{0}\right\rangle$ should be used rather than the inverse of the centroid of the velocity distribution $1 /\left\langle v_{0}\right\rangle$ when applying Eq. (4) in cases with broad velocity distributions [51]. The average velocities vary with mass and atomic number of the fission fragments and need to be determined separately for each nuclide. The centroids of the velocity distributions for the $\mathrm{Y}$ and $\mathrm{Nb}$ isotopes under study are presented in Table I. With velocities of $v_{0} \approx 37 \mu \mathrm{m} / \mathrm{ps}$ and distances between 37 and $1554 \mu \mathrm{m}$, the flight times between the target and the degrader range from 1 to $42 \mathrm{ps}$, indicating approximately the range of lifetimes for which the experiment was sensitive.

\section{RESULTS}

For all odd-mass isotopes of $\mathrm{Y}$ and $\mathrm{Nb}$ under study, the observed $\gamma$-ray spectra are dominated by the transitions within the ground-state band. The $\Delta I=1$ transitions are generally stronger than the $E 2$ crossover transitions and were used to determine the lifetimes as described in the previous section. Specific details of the procedure and the resulting lifetimes for each of the nuclides are presented in the following.

\section{A. ${ }^{99} \mathbf{Y}$}

The total $\gamma$-ray spectrum for ${ }^{99} \mathrm{Y}$, observed with the $90^{\circ}$ detectors and summed for all distances, is shown in Fig. 5. At $90^{\circ}$ the peaks are Doppler broadened due to the finite angular resolution of the Ge detectors, but the deceleration in the degrader foil does not induce any significant Doppler shifts. The $90^{\circ}$ spectrum is therefore well suited to identify the transitions (and potential contaminants) and determine the relative intensities, which are needed to account for the feeding times as discussed in Sec. II B. It is also possible to determine branching ratios for the decay of a given state from this spectrum. However, such branching ratios can be determined

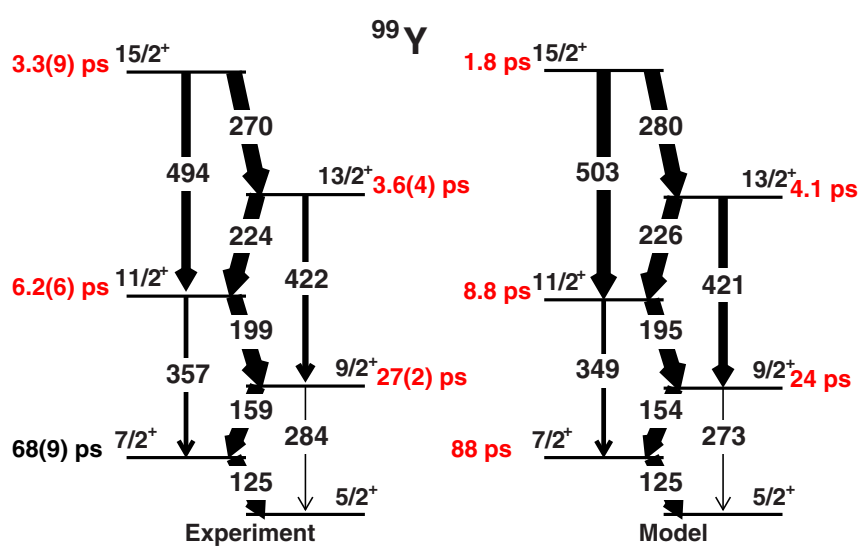

FIG. 6. Partial level scheme for ${ }^{99} \mathrm{Y}$ showing the lowest experimental states and transitions for the $[422] 5 / 2^{+}$ground-state band with the lifetimes measured in this work and a comparison to theoretical calculations using the triaxial particle-rotor model with deformation parameters $\epsilon_{2}=0.41$ and $\gamma=0^{\circ}$. The experimental lifetime for the $7 / 2^{+}$state was taken from Ref. [33]. The widths of the arrows reflect the branching ratios.

with higher precision from $\gamma-\gamma$ coincidence measurements. The branching ratios for the lifetime analysis in ${ }^{99} \mathrm{Y}$ were therefore taken from a coincidence measurement using a ${ }^{252} \mathrm{Cf}$ source [31]. The spectrum for ${ }^{99} \mathrm{Y}$ is entirely dominated by the strongly coupled rotational band built on the $[422] 5 / 2^{+}$ ground state. The band was observed up to spin $19 / 2^{+}$with dominating $\Delta I=1$ transitions and weaker $E 2$ crossover transitions for which the intensity increases with increasing spin. The spectrum shows no indication of transitions originating from the higher-lying three-quasiparticle bands or the known negative-parity states [31]. A partial level scheme for the ground-state band of ${ }^{99} \mathrm{Y}$ is included in Fig. 6, which also shows the results of theoretical particle-rotor calculations as will be discussed in Sect. IV

Figure 7 shows $\gamma$-ray spectra for four $\Delta I=1$ transitions of the ground-state band in ${ }^{99} \mathrm{Y}$ observed at $135^{\circ}$ with respect to the spectrometer axis separately for the seven targetdegrader distances. The intensities of the shifted and unshifted components were determined for each transition by fitting Gaussians with fixed centroids and widths. The intensities yield the so-called decay curves $Q_{i j}(x)$, as described in Sec. II B. The decay curves for the four $\Delta I=1$ transitions in ${ }^{99} \mathrm{Y}$ are shown in Fig. 8. It is apparent from the decay curves that the lifetimes get shorter with increasing spin. Piecewise continuous and differentiable polynomials of second order were fitted to obtain the slope of the decay curves at the various distances. The lifetimes were determined for each distance using the differential decay curve method, where in each case a fraction of the decay curve $Q_{h i}(x)$ of the feeding transitions was subtracted according to the feeding intensities. Only data points with a large slope of the decay curve yield lifetimes with good precision. Due to the relatively large spread in target-degrader distances, typically only two or three data points are within the sensitive region characterized by a large slope of the decay curve (cf. Fig. 8). The final lifetimes were 


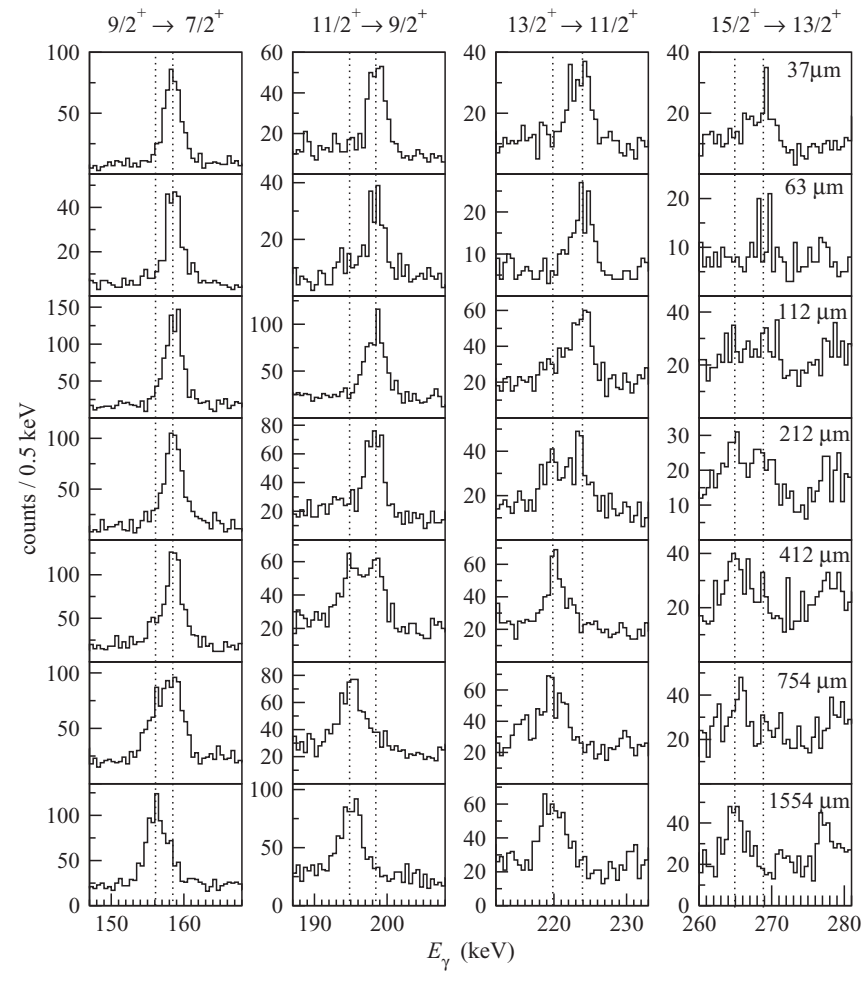

FIG. 7. Spectra showing the $9 / 2^{+} \rightarrow 7 / 2^{+}, 11 / 2^{+} \rightarrow 9 / 2^{+}$, $13 / 2^{+} \rightarrow 11 / 2^{+}$, and $15 / 2^{+} \rightarrow 13 / 2^{+}$transitions in the ground-state band of ${ }^{99} \mathrm{Y}$ observed with the detectors at $135^{\circ}$ with respect to the spectrometer axis for seven target-degrader distances. The spectra are Doppler corrected using the velocity measured in VAMOS ++ after the degrader. The dashed lines indicate the positions of the shifted and unshifted components, respectively.

obtained by taking the weighted mean for the most sensitive data points with large slopes.

Extracting the lifetimes of the $9 / 2^{+}, 11 / 2^{+}$, and $13 / 2^{+}$ states was straightforward, as the decay curves and relative intensities of both the decay and feeding transitions could

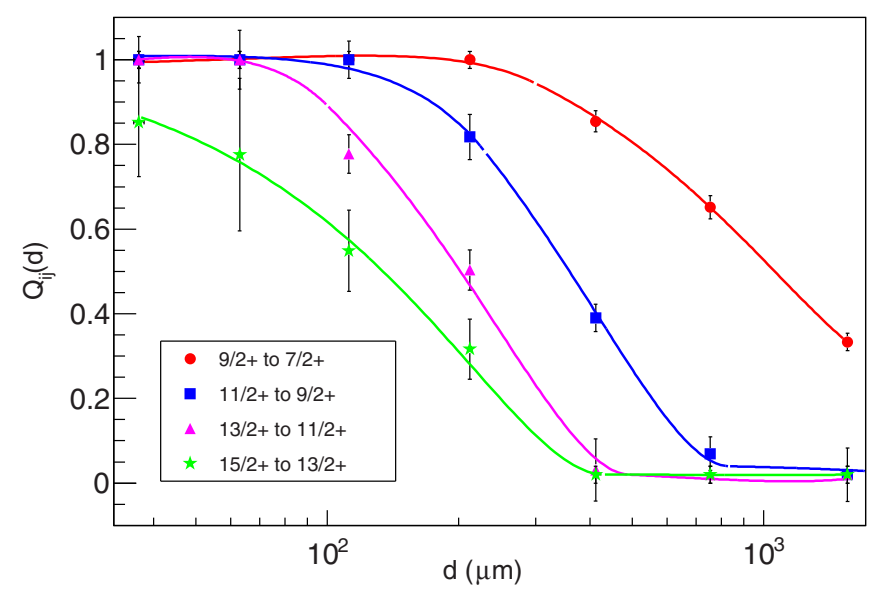

FIG. 8. Decay curves $Q_{i j}$ as a function of the target-degrader distance $d$ for four $\Delta I=1$ transitions in the ground-state band of ${ }^{99} \mathrm{Y}$, as defined in Eq. (3). The lines were obtained by fitting two piecewise continuous and differentiable polynomials of second order and used to determine the slope of the decay curves.

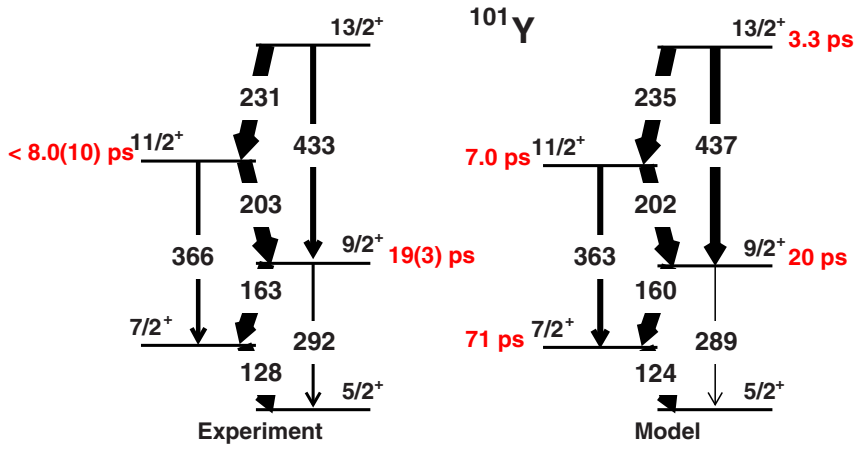

FIG. 9. Partial level scheme for ${ }^{101} \mathrm{Y}$ showing the lowest experimental states and transitions for the $[422] 5 / 2^{+}$ground-state band with the lifetimes measured in this work and a comparison to theoretical calculations using the triaxial particle-rotor model with deformation parameters $\epsilon_{2}=0.43$ and $\gamma=0^{\circ}$.

be measured. The resulting lifetimes are indicated in Fig. 6 . A shifted component of the $125 \mathrm{keV}$ transition from the $7 / 2^{+}$state was observed only for the longest distance. Since furthermore the energy separation between the two components is small due to the low transition energy, it was not possible to determine the lifetime for the $7 / 2^{+}$state. Its lifetime has been measured earlier to be 68(9) ps using fast timing techniques [33]. The $15 / 2^{+}$state is fed from the $17 / 2^{+}$ state by a $284 \mathrm{keV}$ transition which has the same energy as the $9 / 2^{+} \rightarrow 5 / 2^{+}$crossover transition. Since the branching ratio of the 159 and $284 \mathrm{keV}$ transitions from the $9 / 2^{+}$state is known [31] and their decay curves should be identical, it is possible to subtract the contribution of the $9 / 2^{+} \rightarrow 5 / 2^{+}$transition from the total decay curve of the two $284 \mathrm{keV}$ transitions, resulting in the decay curve of the $17 / 2^{+} \rightarrow 15 / 2^{+}$transition, which can be used to account for the feeding time of the $15 / 2^{+}$ state. The resulting lifetime of 3.3(9) ps for the $15 / 2^{+}$state is included in Fig. 6.

\section{B. ${ }^{101} \mathbf{Y}$}

The $\gamma$-ray spectrum observed for ${ }^{101} \mathrm{Y}$ is very similar to the one for ${ }^{99} \mathrm{Y}$, as can be seen in Fig. 5. Because the fission yield for ${ }^{101} \mathrm{Y}$ is low, some strong transitions originating from the isobar ${ }^{101} \mathrm{Zr}$ are visible in the total spectrum observed at $90^{\circ}$. The $\Delta I=1$ transitions of the $[422] 5 / 2^{+}$ground-state band are observed up to spin $17 / 2^{+}$. A partial level scheme showing the experimental energies and transitions for the ground-state band in ${ }^{101} \mathrm{Y}$ is included Fig. 9. The decay curves for the $\Delta I=1$ transitions from the $9 / 2^{+}$and $11 / 2^{+}$states could be extracted from the spectra observed under backward angles at $135^{\circ}$. The spectra for the $9 / 2^{+} \rightarrow 7 / 2^{+}$transition in ${ }^{101} \mathrm{Y}$ for the seven target-degrader distances are included in Fig. 10 to illustrate the quality of the data. For the $7 / 2^{+} \rightarrow 5 / 2^{+}$transition a shifted component was observed only for the longest distance, indicating that the lifetime is long and outside the sensitivity range of the experiment.

The lifetime for the $9 / 2^{+}$state was determined in the same way as for the states in ${ }^{99} \mathrm{Y}$, using the branching ratio from the literature [31] and subtracting the decay curve for the feeding from the $11 / 2^{+}$state, yielding a lifetime of $\tau=19.1(27) \mathrm{ps}$. 


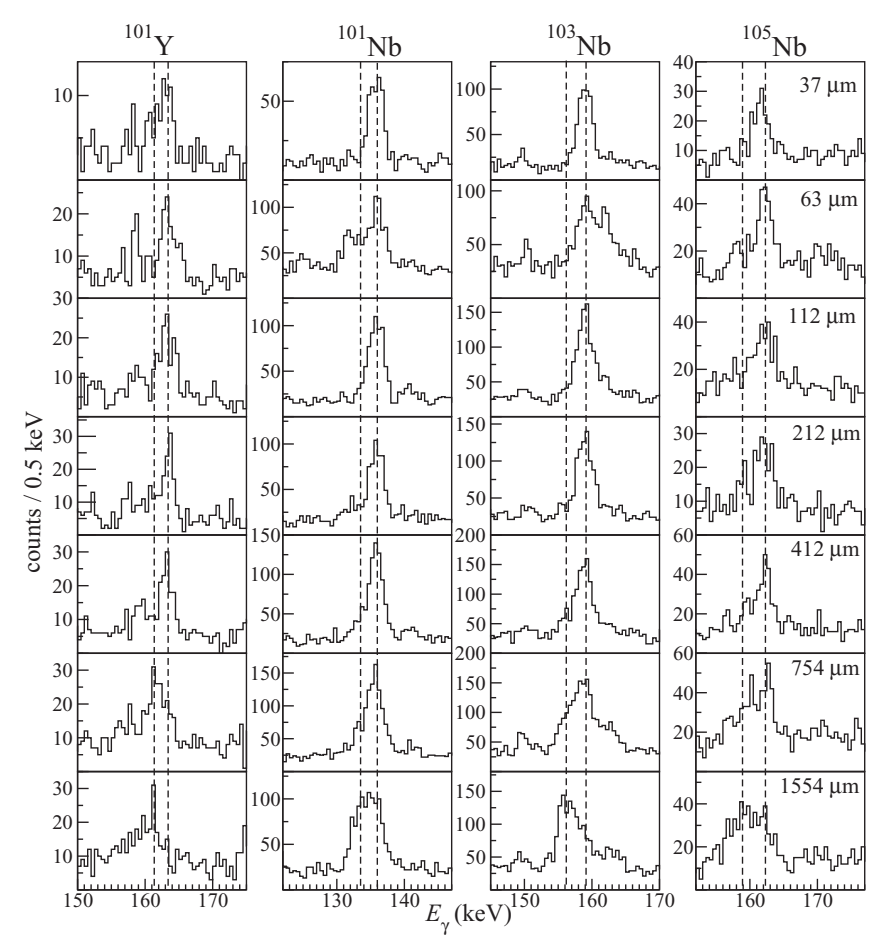

FIG. 10. Spectra showing the $9 / 2^{+} \rightarrow 7 / 2^{+}$transitions in the ground-state bands of ${ }^{101} \mathrm{Y},{ }^{101} \mathrm{Nb},{ }^{103} \mathrm{Nb}$, and ${ }^{105} \mathrm{Nb}$ observed with the detectors at $135^{\circ}$ with respect to the spectrometer axis for the seven target-degrader distances. The spectra are Doppler corrected using the velocity measured in VAMOS ++ after the degrader. The dashed lines indicate the position of the shifted and unshifted components, respectively.

The decay curve for the $13 / 2^{+} \rightarrow 11 / 2^{+}$transition could not be obtained due to lack of statistics. It was consequently not possible to correct the lifetime of the $11 / 2^{+}$state for the feeding from the $13 / 2^{+}$state. Given the similarity to ${ }^{99} \mathrm{Y}$, the lifetime of the $13 / 2^{+}$state is expected to be relatively short with only little influence on determining the lifetime of the $11 / 2^{+}$state. Nevertheless, the result of $\tau=8.0(10)$ ps should be considered an upper limit, because any feeding correction would result in a shorter lifetime.

\section{C. ${ }^{101} \mathrm{Nb}$}

The structure of the odd-mass $\mathrm{Nb}$ isotopes under study is very similar to that of ${ }^{99} \mathrm{Y}$ and ${ }^{101} \mathrm{Y}$, with a strongly coupled rotational band based on the $[422] 5 / 2^{+}$ground state dominating the $\gamma$-ray spectra. The total $\gamma$-ray spectra for ${ }^{101} \mathrm{Nb},{ }^{103} \mathrm{Nb},{ }^{105} \mathrm{Nb}$, and ${ }^{107} \mathrm{Nb}$ obtained with the $90^{\circ}$ detectors are shown in Fig. 11. The $\Delta I=1$ transitions within the ground-state bands carry most of the strength at low spin, while the strength of the $E 2$ crossover transitions increases with spin. For ${ }^{101} \mathrm{Nb}$ the ground-state band was observed up to the $19 / 2^{+}$state. A partial level scheme showing the lowest states and transitions of the ground-state band in ${ }^{101} \mathrm{Nb}$ is included in Fig. 12. In addition to the transitions of the ground-state band the spectrum contains transitions from negative-parity bands based on the [303]5/2- and [301]3/2 $2^{-}$Nilsson orbitals $[31,35]$. The relative strength of the transitions from the

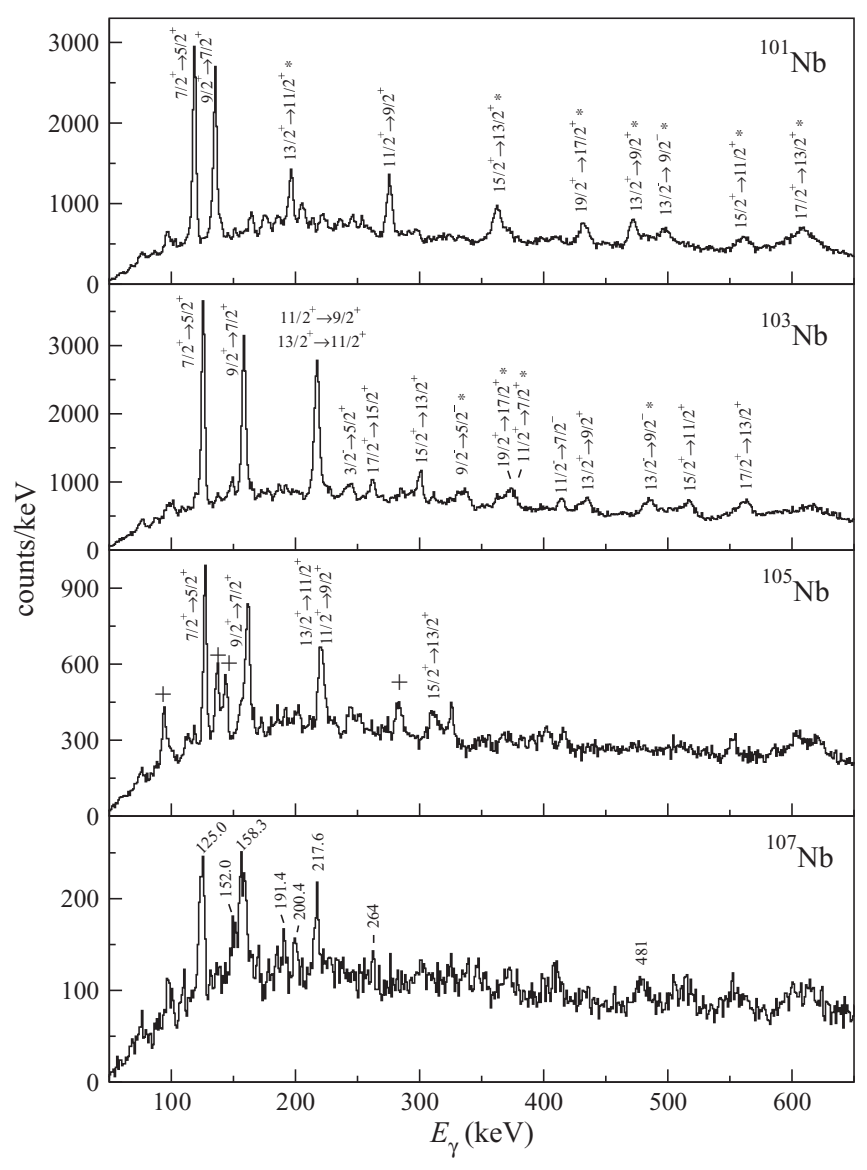

FIG. 11. Total $\gamma$-ray spectra for ${ }^{101} \mathrm{Nb},{ }^{103} \mathrm{Nb},{ }^{105} \mathrm{Nb}$, and ${ }^{107} \mathrm{Nb}$, observed with the $90^{\circ}$ detectors and summed over all distances. Transitions marked with asterisks are suspected to be doublets of similar energy originating from different states in the same nucleus. The transitions labeled with a cross in the spectrum for ${ }^{105} \mathrm{Nb}$ are contaminants originating from ${ }^{105} \mathrm{Mo}$. Transitions in the spectrum for ${ }^{107} \mathrm{Nb}$ are labeled with their energies as no excited states are known for this nucleus.

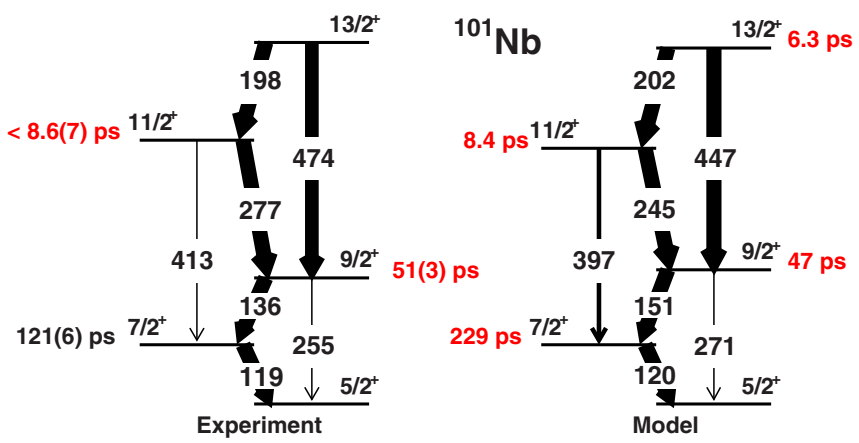

FIG. 12. Partial level scheme for ${ }^{101} \mathrm{Nb}$ showing the lowest experimental states and transitions for the [422]5/2+ ground-state band with the measured lifetimes and a comparison to theoretical calculations using the triaxial particle-rotor model with deformation parameters $\epsilon_{2}=0.35$ and $\gamma=19^{\circ}$. The experimental lifetime for the $7 / 2^{+}$state is taken from a previous fast-timing measurement [40]. 
negative-parity band heads to the ground state is difficult to determine because the states have relatively long lifetimes [40] and decay outside the focus of the EXOGAM detectors. The rotational bands built on the $3 / 2^{-}$and $5 / 2^{-}$states are similar to the ground-state band. Transitions of almost identical energy in the negative-parity bands and the ground-state band [31] make it very difficult to obtain decay curves and relative strengths for some of the transitions. Transitions that are suspected to be doublets are marked with asterisks in Fig. 11 .

The first excited $7 / 2^{+}$state in the ground-state band of ${ }^{101} \mathrm{Nb}$ is known to have a lifetime of 121(6) ps [40], which is outside the sensitivity range of the present experiment. The previous fast-timing measurement found the lifetime of the $9 / 2^{+}$state to be 50(7) ps [40]. From the present data it was possible to obtain a decay curve for the $9 / 2^{+} \rightarrow 7 / 2^{+}$ transition. Spectra from the backward detectors showing this transition are included in Fig. 10. The resulting lifetime was corrected for the $\Delta I=1$ feeding from the $11 / 2^{+}$state. A correction for the $\Delta I=2$ feeding from the $13 / 2^{+}$state was not possible because the $11 / 2^{-} \rightarrow 7 / 2^{-}$transition in the [301]3/2- band has the same energy [31]. As the lifetime of the $13 / 2^{+}$state is expected to be significantly shorter compared to the $9 / 2^{+}$state, the influence from the $\Delta I=2$ feeding is expected to be negligible. The lifetime of the $9 / 2^{+}$ state is found to be 50.7(30) ps. The excellent agreement with the previously measured value suggests that systematic uncertainties are insignificant in the present measurement.

The present data also yield a decay curve for the $11 / 2^{+} \rightarrow$ $9 / 2^{+}$transition. However, the feeding $13 / 2^{+} \rightarrow 11 / 2^{+}$transition at $197 \mathrm{keV}$ is a doublet with the $9 / 2^{-} \rightarrow 7 / 2^{-}$transition at $198 \mathrm{keV}$ in the [303]5/2- band [31]. Since the $9 / 2^{-} \rightarrow 5 / 2^{-}$ transition also is a doublet with the $15 / 2^{+} \rightarrow 13 / 2^{+}$transition, it is not possible to disentangle the feeding contribution from the $13 / 2^{+} \rightarrow 11 / 2^{+}$transition. It was therefore not possible to correct the lifetime of the $11 / 2^{+}$state for feeding, and only an upper limit of $\tau \leqslant 8.6(7)$ ps could be extracted.

\section{D. ${ }^{103} \mathrm{Nb}$}

Also in ${ }^{103} \mathrm{Nb}$ the ground-state band is built on the [422]5/2 $2^{+}$Nilsson orbital. Previous experiments identified negative-parity bands built on the $[303] 5 / 2^{-}$and $[301] 3 / 2^{-}$ Nilsson states [34] and candidates for one-phonon and twophonon $\gamma$-vibrational bands [37]. The total $\gamma$-ray spectrum for ${ }^{103} \mathrm{Nb}$ observed at $90^{\circ}$ is shown in Fig. 11. The groundstate band is observed up to spin $17 / 2^{+}$. The spectrum also shows the $3 / 2^{-} \rightarrow 5 / 2_{g s}^{+}$transition from the $[301] 3 / 2^{-}$band head and several transitions originating from the [303]5/2band, which is more strongly populated. The $5 / 2^{-}$and $3 / 2^{-}$ band heads have long lifetimes of 7.36(14) and 1.80(6) ns [41], respectively. As a consequence the transitions occur far from the collimated EXOGAM detectors and their observed intensities are low. In the present experiment lifetimes could only be obtained for levels in the ground-state band.

The $\Delta I=1$ transitions are dominant in the ground-state band, although the relative strength of the $\Delta I=2$ transition increases at higher spin. A partial level scheme showing the lowest states and transitions of the ground-state band is included in Fig. 13. The $7 / 2^{+}$state has a known lifetime of

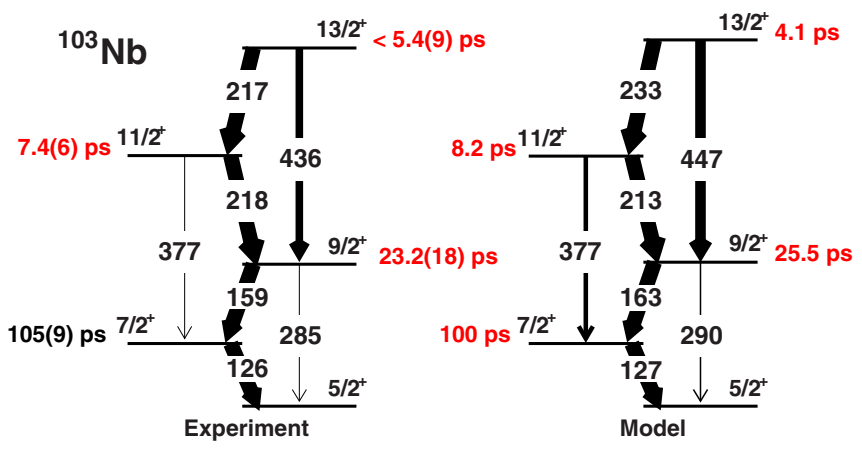

FIG. 13. Partial level scheme for ${ }^{103} \mathrm{Nb}$ showing the lowest experimental states and transitions for the $[422] 5 / 2^{+}$ground-state band with the measured lifetimes and a comparison to theoretical calculations using the triaxial particle-rotor model with deformation parameters $\epsilon_{2}=0.37$ and $\gamma=15^{\circ}$. The experimental lifetime for the $7 / 2^{+}$state is taken from a previous fast-timing measurement [41].

105(9) ps [41], which is outside of the sensitive range of the present experiment. Spectra for the $9 / 2^{+} \rightarrow 7 / 2^{+}$transition are included in Fig. 10, showing the shifted and unshifted components of the transition at different distances. The two consecutive $13 / 2^{+} \rightarrow 11 / 2^{+}$and $11 / 2^{+} \rightarrow 9 / 2^{+}$transitions have nearly identical energy of 218 and $217 \mathrm{keV}$, respectively, making it difficult to correct the lifetime of the $9 / 2^{+}$state for their feeding contribution. However, it was possible to obtain the decay curve for the $436 \mathrm{keV} 13 / 2^{+} \rightarrow 9 / 2^{+}$transition. The decay curve from this transition can be used, first, to determine the lifetime of the $13 / 2^{+}$state, but also, with known branching ratios, to correct for the contribution of the $13 / 2^{+} \rightarrow 11 / 2^{+}$transition in the total $217 / 218 \mathrm{keV}$ peak. With branching ratios given in the literature [52], the estimated strength of the $13 / 2^{+} \rightarrow 11 / 2^{+}$transition from the observed strength of the $435 \mathrm{keV}$ peak is much too large compared to the total strength of the $217 / 218 \mathrm{keV}$ peak. Instead of using a branching ratio that might be unreliable because of the doublet, the relative strength of the 217 and $218 \mathrm{keV}$ cascade was estimated by assuming that the total population of the states within the band as a function of excitation energy is identical to that of the ground-state bands in neighboring $\mathrm{Y}$ isotopes, which all show a linear decrease above the $9 / 2^{+}$ state. Using this assumption, the populations of the $11 / 2^{+}$and $13 / 2^{+}$states were interpolated between the $9 / 2^{+}$and $15 / 2^{+}$ states and found to be $80(5) \%$ and $60(5) \%$ relative to the $9 / 2^{+}$ state, respectively, which is in very good agreement with the observed total intensity of the $217 / 218 \mathrm{keV}$ peak. The resulting $(\Delta I=2) /(\Delta I=1)$ branching ratio for the $13 / 2^{+}$state of $52(8) \%$ is larger than the literature value of $18 \%$ [52], but in much better agreement with the systematic trend observed in neighboring isotopes.

Knowing the relative intensities of the transitions in the ground-state band of ${ }^{103} \mathrm{Nb}$, it is now possible to disentangle their decay curves $Q(x)$. The decay curve for the $13 / 2^{+} \rightarrow$ $11 / 2^{+}$transition can be assumed to be identical to the known decay curve of the $13 / 2^{+} \rightarrow 9 / 2^{+}$transition. The decay curve of the $11 / 2^{+} \rightarrow 9 / 2^{+}$transition can then be obtained by subtracting the decay curve of the $13 / 2^{+} \rightarrow 9 / 2^{+}$transition 
from the total measured decay curve for the unresolved $217 / 218 \mathrm{keV}$ peak, weighted by the relative intensity of the $13 / 2^{+} \rightarrow 11 / 2^{+}$transition. The lifetime of the $9 / 2^{+}$state is found to be 23.1(18) ps by evaluating the decay curve of the $9 / 2^{+} \rightarrow 7 / 2^{+}$transition and subtracting the feeding from both the $11 / 2^{+}$and $13 / 2^{+}$states. With the decay curve for the $11 / 2^{+} \rightarrow 9 / 2^{+}$transition obtained as described above and subtracting the feeding from the $13 / 2^{+}$state, the lifetime of the $11 / 2^{+}$state is found to be 7.4(6) ps. Finally, the lifetime of the $13 / 2^{+}$state is obtained from the decay curve of the $13 / 2^{+} \rightarrow 9 / 2^{+}$transition to be 5.4(9) ps. However, because the decay curve of the $15 / 2^{+} \rightarrow 13 / 2^{+}$feeding transition could not be determined, the lifetime of the $13 / 2^{+}$state should be considered an upper limit.

For further analysis of the lifetime results it is desirable to find the branching ratio for the decay of the $9 / 2^{+}$state, which is not given in the literature. Its determination is not straightforward because the $285 \mathrm{keV} 9 / 2^{+} \rightarrow 5 / 2^{+}$transition is a doublet with the $7 / 2_{2}^{-} \rightarrow 3 / 2_{1}^{-}$transition in the [301]3/2band [53]. Since the branching ratio for the $7 / 2_{2}^{-}$state is known [52], the contribution of the $7 / 2_{2}^{-} \rightarrow 3 / 2_{1}^{-}$transition to the $286 \mathrm{keV}$ peak can be subtracted by measuring the intensity of the $166 \mathrm{keV} 7 / 2_{2}^{-} \rightarrow 5 / 2_{2}^{-}$transition. The latter can be assumed to be pure because the $164 \mathrm{keV} 5 / 2_{1}^{-} \rightarrow 5 / 2_{g s}^{+}$ transition occurs outside the focus of EXOGAM due to the long lifetime of the $5 / 2_{1}^{-}$state of 7.36(14) ns [41]. In this way a $(\Delta I=2) /(\Delta I=1)$ branching ratio of $6(4) \%$ is obtained for the $9 / 2^{+}$state.

\section{E. ${ }^{105} \mathrm{Nb}$}

The assignment of a rotational band observed following spontaneous fission of ${ }^{248} \mathrm{Cm}$ as the [422]5/2+ ground-state band in ${ }^{105} \mathrm{Nb}$ was based on its similarity with the ground-state band in ${ }^{103} \mathrm{Nb}$ and correlations with transitions in various isotopes of the complementary Cs fission fragments [36]. The band was later extended up to spin $25 / 2^{+}$following spontaneous fission of ${ }^{252} \mathrm{Cf}$ [31]. Also candidates for multiphonon $\gamma$ vibrational bands were identified [38], whereas negative-parity bands have not yet been observed. No lifetimes of excited states in ${ }^{105} \mathrm{Nb}$ were previously measured. In the present experiment $\Delta I=1$ transitions in the ground-state band were observed up to spin $15 / 2^{+}$, confirming its assignment to ${ }^{105} \mathrm{Nb}$. The spectrum observed at $90^{\circ}$, summed over all distances, is shown in Fig. 11. Some of the strongest transitions originating from the isobar ${ }^{105} \mathrm{Mo}$ are visible in the spectrum as contaminants due to finite resolution in atomic number $Z$. Some transitions that are visible in the spectrum remain unidentified. It may be speculated that some of them originate from negative-parity states, but without $\gamma-\gamma$ coincidences it is difficult to extend the level scheme. A partial level scheme for the ground-state band of ${ }^{105} \mathrm{Nb}$ is included in Fig. 14 .

The spectra showing the $9 / 2^{+} \rightarrow 7 / 2^{+}$transition at different target-degrader distances are included in Fig. 10. From these spectra it is straightforward to obtain the decay curve for the $9 / 2^{+} \rightarrow 7 / 2^{+}$transition. Also in ${ }^{105} \mathrm{Nb}$ the $\Delta I=1$ transitions from the $11 / 2^{+}$and $13 / 2^{+}$states have similar energies of $220.6 \mathrm{keV}$ and $223.4 \mathrm{keV}$, respectively. The energy difference between these two transitions of $2.8 \mathrm{keV}$

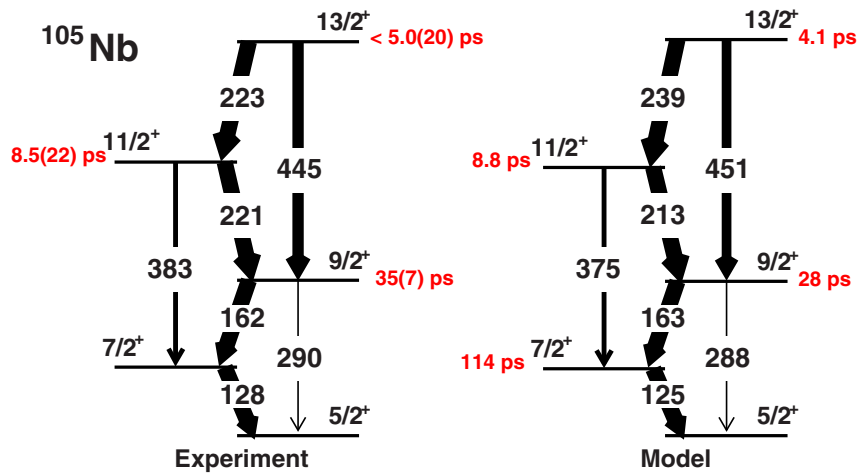

FIG. 14. Partial level scheme for ${ }^{105} \mathrm{Nb}$ showing the lowest experimental states and transitions for the $[422] 5 / 2^{+}$ground-state band with the measured lifetimes and a comparison to theoretical calculations using the triaxial particle-rotor model with deformation parameters $\epsilon_{2}=0.36$ and $\gamma=13^{\circ}$.

is sufficiently large to separate the two full energy peaks. However, the shifted component of the $223.4 \mathrm{keV}$ transition overlaps with the unshifted component of the $220.6 \mathrm{keV}$ transition. From the $\gamma$-ray spectra it is possible to obtain the intensity of the shifted component of the $220.6 \mathrm{keV} \mathrm{11/2^{+ } \rightarrow}$ $9 / 2^{+}$transition, $J_{11 / 2}^{s}(x)$, the unshifted component of the 223.4 $\mathrm{keV} 13 / 2^{+} \rightarrow 11 / 2^{+}$transition, $J_{13 / 2}^{u}(x)$, and the sum of the overlapping components, $J_{11 / 2}^{u}+J_{13 / 2}^{s}$. Because the relative strength of the two transitions, $\left(J_{13 / 2}^{u}+J_{13 / 2}^{s}\right) /\left(J_{11 / 2}^{u}+J_{11 / 2}^{s}\right)$, is the same for all distances, it is possible to disentangle the two overlapping components and determine the intensities for all four components. For the longest distance both states decay before reaching the degrader and the full strength appears in the shifted component for both transitions, without any sign of an unshifted peak. The spectrum obtained for the longest distance can therefore be used to determine the relative strength of the two transitions. The strength of the $223.4 \mathrm{keV}$ transitions relative to the $220.6 \mathrm{keV}$ transition is found to be $51 \%$, which is in very good agreement with the previously reported value of $49 \%$ [31]. Using the same relative strength of the two transitions for all distances it was possible to obtain decay curves also for the $13 / 2^{+} \rightarrow 11 / 2^{+}$and $11 / 2^{+} \rightarrow 9 / 2^{+}$ transitions. The resulting lifetimes are 34.9(66) ps for the $9 / 2^{+}$ state, $8.5(22) \mathrm{ps}$ for $11 / 2^{+}$state, and 5.0(20) ps for the $13 / 2^{+}$ state. Because it was impossible to obtain a decay curve for the $15 / 2^{+} \rightarrow 13 / 2^{+}$transition, the feeding time into the $13 / 2^{+}$ state was not accounted for and the resulting lifetime of the $13 / 2^{+}$state should be considered an upper limit.

$$
\text { F. }{ }^{107} \mathrm{Nb}
$$

To our knowledge, no information on excited states in ${ }^{107} \mathrm{Nb}$ has been published prior to this work. The half-life of the ground state was measured to be 280(20) ms [54,55], but the spin-parity of the ground-state is unknown. The total spectrum from the $90^{\circ}$ detectors obtained in coincidence with fission fragments identified as ${ }^{107} \mathrm{Nb}$ is shown in Fig. 11. Because the fission yield for ${ }^{107} \mathrm{Nb}$ is very low, care has to be taken to exclude that the transitions originate from misidentified fission fragments that were produced with higher yield. In the 
case of ${ }^{101} \mathrm{Y}$ and ${ }^{105} \mathrm{Nb}$ the spectra were contaminated by the $Z+1$ isobars ${ }^{101} \mathrm{Zr}$ and ${ }^{105} \mathrm{Mo}$, respectively. The strongest transitions in the spectrum gated on ${ }^{107} \mathrm{Nb}$, however, cannot be explained by transitions in ${ }^{107}$ Mo. The spectrum shown in Fig. 11 does not resemble the spectrum that is obtained when gating on fission fragments identified as ${ }^{107} \mathrm{Mo}$, which is dominated by $E 2$ transitions. Only the peak observed at 152 $\mathrm{keV}$ could potentially be due to the relatively strong $7 / 2^{+} \rightarrow$ $5 / 2^{+}$transition in ${ }^{107}$ Mo.

Also the possibility of a misidentification of the mass in VAMOS ++ has to be considered. Transitions in the odd-odd nuclei ${ }^{104} \mathrm{Nb}$ [56] and ${ }^{106} \mathrm{Nb}$ [57] have been identified following spontaneous fission by gating on the complementary La fission partners. The spectrum of the $\mathrm{Nb}$ isotopes with even mass is quite different from those with odd mass, and none of the known transitions in ${ }^{104} \mathrm{Nb}$ and ${ }^{106} \mathrm{Nb}$ could explain the peaks observed in coincidence with ${ }^{107} \mathrm{Nb}$. The spectrum for ${ }^{107} \mathrm{Nb}$ resembles that of ${ }^{105} \mathrm{Nb}$, but the energies of the three strongest transitions are approximately $4 \mathrm{keV}$ different from the first three $\Delta I=1$ transitions in ${ }^{105} \mathrm{Nb}$. As a consequence, the spectrum for ${ }^{107} \mathrm{Nb}$ cannot be explained by contaminations from ${ }^{105} \mathrm{Nb}$. The transitions at 158.3(5) and 217.6(5) keV in the spectrum for ${ }^{107} \mathrm{Nb}$ are very similar in energy to the $9 / 2^{+} \rightarrow$ $7 / 2^{+}$and $11 / 2^{+} \rightarrow 9 / 2^{+}$transitions in ${ }^{103} \mathrm{Nb}$, which have energies of 158.9 and $218.3 \mathrm{keV}$, respectively. The transition at $125.0(5) \mathrm{keV}$, on the other hand, is clearly different from the $7 / 2^{+} \rightarrow 5 / 2^{+}$transition at $126.4 \mathrm{keV}$ in ${ }^{103} \mathrm{Nb}$. The most probable charge states for the $\mathrm{Nb}$ fission fragments are $q=$ $29^{+}, 30^{+}$, and $31^{+}$. Fragments of mass $A=107$ and charge state $q$ have, consequently, a mass-over-charge ratio similar to that of fragments with mass $A=103$ and charge state $(q-1)$. The scenario in which the transitions observed in coincidence with ${ }^{107} \mathrm{Nb}$ are explained by transitions originating from ${ }^{103} \mathrm{Nb}$ therefore cannot be ruled out completely. Nevertheless, with a difference of four mass units and a significant difference in $\gamma$-ray energy for at least one of the three strongest transitions, it is most likely that the observed transitions originate indeed from ${ }^{107} \mathrm{Nb}$ and correspond to the first three $\Delta I=1$ transitions of the ground-state band. This suggests that the structure of ${ }^{107} \mathrm{Nb}$ is very similar compared to the lighter odd-mass isotopes with a strongly coupled rotational band built on a $5 / 2^{+}$ ground state. Without sufficient data for $\gamma-\gamma$ coincidences it is difficult to assign any of the other transitions that are visible in the spectrum, for example at 191.4(5) and 200.4(5) keV. A relatively strong transition is observed at $481 \mathrm{keV}$, which corresponds to the sum of the $217 \mathrm{keV}$ transition and another weaker transition at $264 \mathrm{keV}$. These two transitions are therefore candidates for transitions depopulating the $13 / 2^{+}$ state. The suggested level scheme is shown in Fig. 15. The amount of data for ${ }^{107} \mathrm{Nb}$ is unfortunately insufficient to determine any lifetimes.

\section{DISCUSSION}

The low-lying structure of the $\mathrm{Y}$ and $\mathrm{Nb}$ isotopes with $N \geqslant$ 60 is dominated by the strongly coupled rotational bands built on the $[422] 5 / 2^{+}$ground state. The neighboring even-even isotopes of $\mathrm{Sr}, \mathrm{Zr}$, and $\mathrm{Mo}$ are well deformed, and the particlerotor model is an obvious choice to describe the properties
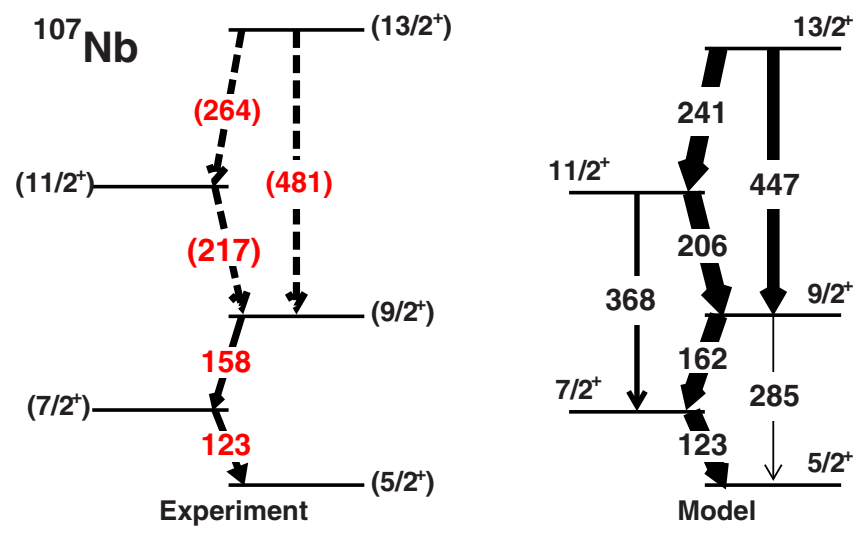

FIG. 15. Suggested level scheme for ${ }^{107} \mathrm{Nb}$ based on the strongest transitions observed in coincidence with fission fragments identified as ${ }^{107} \mathrm{Nb}$. The transitions shown with dashed arrows are only tentative. The experimental level scheme is compared to theoretical calculations using the triaxial particle-rotor model with deformation parameters $\epsilon_{2}=0.37$ and $\gamma=9^{\circ}$.

of the odd-mass nuclei with an unpaired proton. The energies of the states in the rotational bands were indeed found to be well reproduced by a rigid triaxial particle-rotor model [31]. The lifetimes measured in the present work allow extracting electromagnetic transition probabilities, which provide a more rigorous benchmark for theoretical models and quantitative information on the underlying nuclear shapes.

\section{A. Simple rigid rotor model}

In its simplest form, the particle-rotor model assumes a rigid, axially deformed core described by a constant intrinsic quadrupole moment $Q_{0}$. The reduced transition probabilities for $E 2$ and $M 1$ transition within a rotational band are then found as [58]

$$
\begin{aligned}
B\left(E 2 ; I_{i} \rightarrow I_{f}\right) & =\frac{5}{16 \pi} e^{2} Q_{0}^{2}\left|\left\langle I_{i} 2 K 0 \mid I_{f} K\right\rangle\right|^{2}, \\
B\left(M 1 ; I_{i} \rightarrow I_{f}\right) & =\frac{3}{4 \pi} \mu_{K}^{2}\left(g_{K}-g_{R}\right)^{2} K^{2}\left|\left\langle I_{i} 1 K 0 \mid I_{f} K\right\rangle\right|^{2},
\end{aligned}
$$

where $g_{K}$ and $g_{R}$ are the gyromagnetic factors of the particle and core, respectively, and the expression for $M 1$ transitions is valid for spin projections $K \neq 1 / 2$. Within this simple model the ratio of $g$ factors and quadrupole moment, $\left(g_{K}-g_{R}\right) / Q_{0}$, is easily obtained from the branching ratio $b$ between the $\Delta I=$ 1 and $\Delta I=2$ transitions,

$$
b=\frac{T(E 2)_{\Delta I=2}}{T(M 1)_{\Delta I=1}+T(E 2)_{\Delta I=1}}=\frac{I_{\gamma, \Delta I=2}}{I_{\gamma, \Delta I=1}},
$$

where $T(\sigma \lambda)$ are transition probabilities and $I_{\gamma}$ the intensities of the $\gamma$-ray transitions. Combining the well-known branching ratios with the newly measured lifetimes, it is now possible to extract $\left(g_{K}-g_{R}\right)$ and $Q_{0}$ independently. Using the model assumptions of Eqs. (5) and (6) and the results for $\left(g_{K}-g_{R}\right)$ and $Q_{0}$ it is then furthermore possible to extract the $E 2 / M 1$ 
TABLE II. E2/M1 mixing ratios, $g$ factors, and intrinsic quadrupole moments extracted from the experimental lifetimes and branching ratios using the model assumptions of Eqs. (5) and (6).

\begin{tabular}{ccccc}
\hline \hline & $I_{i}$ & $\delta^{2}(E 2 / M 1)$ & $g_{K}-g_{R}$ & $Q_{0}(e \mathrm{~b})$ \\
\hline \multirow{6}{*}{${ }^{99} \mathrm{Y}$} & $9 / 2^{+}$ & $0.030(2)$ & $0.97_{-0.03}^{+0.04}$ & $5.00_{-0.22}^{+0.23}$ \\
& $11 / 2^{+}$ & $0.026(2)$ & $1.25_{-0.06}^{+0.07}$ & $5.90_{-0.30}^{+0.34}$ \\
& $13 / 2^{+}$ & $0.016(1)$ & $1.27_{-0.05}^{+0.06}$ & $4.96_{-0.23}^{+0.25}$ \\
& $15 / 2^{+}$ & $0.018(2)$ & $0.92_{-0.11}^{+0.16}$ & $3.65_{-0.43}^{+0.65}$ \\
& $9 / 2^{+}$ & $0.038(3)$ & $1.08_{-0.07}^{+0.09}$ & $6.07_{-0.44}^{+0.53}$ \\
${ }^{101} \mathrm{Y}$ & $11 / 2^{+}$ & $0.028(2)$ & $1.05_{-0.07}^{+0.07}$ & $5.06_{-0.31}^{+0.37}$ \\
& $9 / 2^{+}$ & $0.014(2)$ & $0.93(3)$ & $3.79(22)$ \\
${ }^{101} \mathrm{Nb}$ & $11 / 2^{+}$ & $0.037(4)$ & $0.68(3)$ & $2.75(17)$ \\
& $9 / 2^{+}$ & $0.010(7)$ & $1.11(5)$ & $3.26_{-1.37}^{+0.89}$ \\
${ }^{103} \mathrm{Nb}$ & $11 / 2^{+}$ & $0.008(4)$ & $1.12_{-0.05}^{+0.06}$ & $2.66_{-0.66}^{+0.52}$ \\
& $13 / 2^{+}$ & $0.014(3)$ & $1.07_{-0.09}^{+0.11}$ & $3.93_{-0.40}^{+0.45}$ \\
& $9 / 2^{+}$ & $0.029(3)$ & $0.83_{-0.07}^{+0.09}$ & $4.11_{-0.39}^{+0.50}$ \\
${ }^{105} \mathrm{Nb}$ & $11 / 2^{+}$ & $0.034(3)$ & $0.90_{-0.10}^{+0.15}$ & $4.38_{-0.49}^{+0.73}$ \\
& $13 / 2^{+}$ & $0.021(2)$ & $0.98_{-0.15}^{+0.29}$ & $4.40_{-0.68}^{+1.29}$ \\
\hline \hline
\end{tabular}

mixing ratio of the $\Delta I=1$ transitions,

$$
\delta^{2}(E 2 / M 1)=\frac{T(E 2)_{\Delta I=1}}{T(M 1)_{\Delta I=1}} .
$$

The mixing ratios, $g$ factors, and quadrupole moments extracted using this simple model are presented in Table II. The quoted uncertainties are propagated from the experimental uncertainties of the lifetimes and branching ratios. It should be noted, however, that the results are strongly model dependent.

The mixing ratios are found to be very small, indicating that the $\Delta I=1$ transitions are dominated by their $M 1$ component with only a very small $E 2$ contribution. The quadrupole moments are large and to good approximation constant, which suggests that it is not unreasonable to apply this simple particle-rotor model to the ground-state bands. It is observed that the quadrupole moments for the $\mathrm{Y}$ isotopes are larger than those for the $\mathrm{Nb}$ isotopes. This observation is consistent with the measured spectroscopic quadrupole moments for the ground states of ${ }^{99,101} \mathrm{Y}$ [32] and ${ }^{101,103} \mathrm{Nb}$ [39], which show the same trend. Using the relation between intrinsic and spectroscopic quadrupole moments of the rotational model,

$$
Q_{s}=\frac{3 K^{2}-I(I+1)}{(I+1)(2 I+3)} Q_{0},
$$

one obtains $Q_{0}=4.3(5) e b$ from the measured spectroscopic quadrupole moments for the ground states in ${ }^{99} \mathrm{Y}$ and ${ }^{101} \mathrm{Y}$, and $Q_{0}=3.0(2) e b$ for ${ }^{101} \mathrm{Nb}$ and ${ }^{103} \mathrm{Nb}$. These values are similar to the results obtained for the excited states with the simple particle-rotor model. The $\left(g_{K}-g_{R}\right)$ values are also more or less constant within the bands and similar for all nuclides under study. For a proton in a $g_{9 / 2}$ orbital one expects $g_{K} \approx 1.5$, and approximating $g_{R} \approx Z / A \approx 0.4$, the values of $\left(g_{K}-g_{R}\right) \approx$ 1.1 are consistent with the presumed $[422] 5 / 2^{+}$configuration. These $g$ factors are furthermore consistent with the measured magnetic moments for the ground states in ${ }^{99,101} \mathrm{Y}$ [32] and
${ }^{101,103} \mathrm{Nb}$ [39]. The measured value of $\mu \approx 3.2 \mu_{N}$ for all four nuclides corresponds to $\left(g_{K}-g_{R}\right) \approx 1.2$.

\section{B. Reduced transition probabilities}

The in-band decay is described by the reduced transition probabilities $B(M 1 ; \Delta I=1), B(E 2 ; \Delta I=1)$, and $B(E 2 ; \Delta I=2)$. To determine all three transition probabilities for a given state model independently, the $E 2 / M 1$ mixing ratio of the $\Delta I=1$ transition is needed as a third observable in addition to the branching ratio and lifetime. This quantity can in principle be measured, e.g., from angular distributions, but in practice it is very difficult to obtain precise values. With limited statistics and limited angular coverage of the clover detectors it was not possible to measure reliable mixing ratios in the present experiment, and these quantities remain unknown for the transitions in question.

The three transition probabilities can nevertheless be extracted if one assumes that each state is characterized by a quadrupole moment $Q_{0}$ which determines the strengths of the two $E 2$ transitions with $\Delta I=1$ and $\Delta I=2$. In that case the $B(E 2 ; \Delta I=1)$ and $B(E 2 ; \Delta I=2)$ values are related by Clebsch-Gordan coefficients as

$$
\frac{B(E 2 ; I \rightarrow I-1)}{B(E 2 ; I \rightarrow I-2)}=\left|\frac{\langle I 2 K 0 \mid(I-1) K\rangle}{\langle I 2 K 0 \mid(I-2) K\rangle}\right|^{2} .
$$

It should be noted that this assumption is far less restrictive than that of a constant intrinsic quadrupole moment for the entire rotational band and introduces only a weak model dependence of the $B(M 1)$ and $B(E 2)$ values. The three transition probabilities that reproduce for each state the experimental lifetime and branching ratio and their uncertainties, with the additional constraint that Eq. (10) is fulfilled, are presented in Table III and Fig. 16.

\section{Triaxial particle-rotor calculations}

To interpret the measured transition probabilities and draw conclusions about the underlying nuclear shapes, the results are compared to triaxial particle-rotor calculations [59]. Single-particle energies and eigenfunctions were calculated in a triaxially deformed modified harmonic oscillator potential for fixed quadrupole deformations $\left(\epsilon_{2}, \gamma\right)$ using standard Nilsson parameters [60]. Particle-rotor states were then constructed by diagonalizing the particle-rotor Hamiltonian in the strong coupling basis with up to 15 Nilsson orbitals [61]. The residual pairing interaction was treated within the BCS approximation with standard values for the pairing strength [62]. The moment of inertia parameters for the three axes of the triaxial core were assumed to be related by a hydrodynamical expression and were determined from the energy of the first $2^{+}$state of the even-even core nucleus. As the Coriolis coupling between Nilsson states is often found to be too large [63], an attenuation factor $\xi$ is introduced to reduce the off-diagonal matrix elements. In the final step electromagnetic matrix elements were calculated from the particle-rotor eigenstates. For magnetic transitions the intrinsic $g_{s}$ factor can be reduced from the free value by introducing a scaling factor $n_{g s}$, whereas the $g$ factor of the core was approximated as $g_{R}=Z / A$. 
TABLE III. Experimental and theoretical lifetimes $(\tau)$, branching ratios $(b)$, transition energies $\left(E_{\gamma}\right)$, and reduced transition probabilities for various states in ${ }^{99} \mathrm{Y},{ }^{101} \mathrm{Y},{ }^{101} \mathrm{Nb},{ }^{103} \mathrm{Nb}$, and ${ }^{105} \mathrm{Nb}$. The experimental reduced transition probabilities were obtained by assuming that the $E 2$ strengths are related by angular momentum coupling coefficients as shown in Eq. (10). The theoretical values were obtained with the triaxial particle-rotor model based on a modified harmonic oscillator potential. (See text for details.)

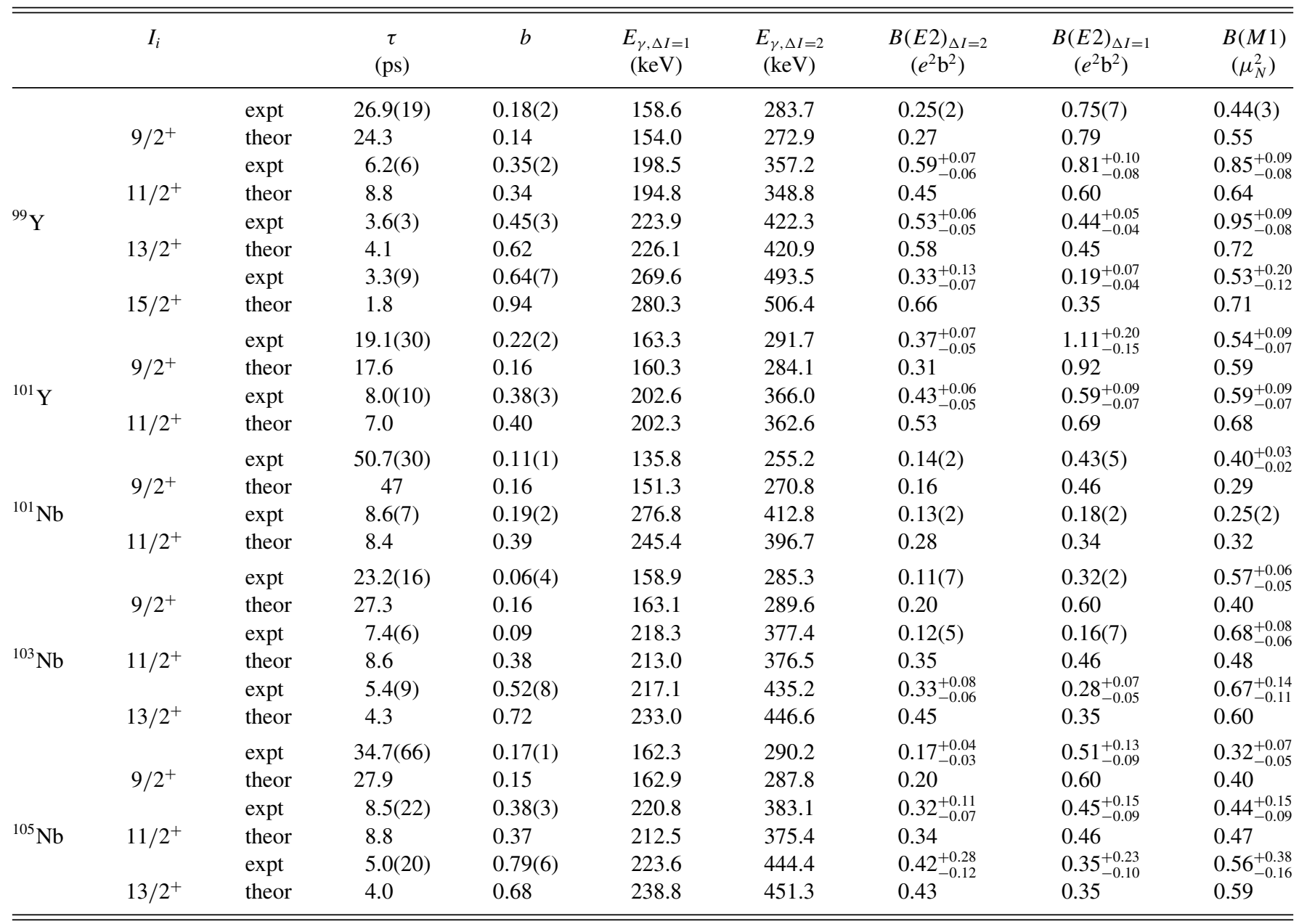

For the comparison of the experimental results with the particle-rotor calculations the following iterative procedure was chosen: In a first step the deformation parameters $\epsilon_{2}$ and $\gamma$ were adjusted to reproduce the energies of the states in the [422]5/2+ ground-state band. The triaxiality parameter $\gamma$ is largely determined by the signature splitting of the band. In some cases the fit could be improved by varying the moment of inertia parameters by slightly modifying the value obtained from the energy of the first $2^{+}$state from the experimental value in the $\mathrm{Sr}$ and $\mathrm{Zr}$ core nuclei. If necessary also the Coriolis attenuation factor $\xi$ was adjusted, but the use of this parameter was avoided if possible. In the next step the electromagnetic transition probabilities were calculated. In some cases a modest scaling of the $g_{s}$ factor was introduced to better reproduce the experimental $E 2 / M 1$ branching ratios. This procedure was iterated until a satisfactory fit of the energies and branching ratios in the ground-state band was achieved. Finally the lifetimes of the states were calculated from the transition probabilities. The five parameters of the model are shown Table IV. The four parameters relevant for the energies of the states are very similar to those chosen in the previous work of Luo et al. [31]. The calculated energies of the states are compared to the experimental values in Fig. 6, $9,12,13,14$, and 15 for all $\mathrm{Y}$ and $\mathrm{Nb}$ isotopes under study. The calculated lifetimes and branching ratios are compared to the experimental values in Table III, which also includes a comparison for the reduced transition probabilities. The experimental and calculated $B(E 2 ; I \rightarrow I-2), B(E 2 ; I \rightarrow$ $I-1)$, and $B(M 1 ; I \rightarrow I-1)$ values are plotted in Fig. 16 .

In general the triaxial particle-rotor model gives a very satisfactory description of the energies and transition probabilities for the ground-state bands of the $\mathrm{Y}$ and $\mathrm{Nb}$ isotopes with $N \geqslant 60$. The measured lifetimes and their comparison to particle-rotor calculations support the earlier conclusion of Luo et al. [31] that the ground-state bands in both ${ }^{99} \mathrm{Y}$ and ${ }^{101} \mathrm{Y}$ are well reproduced by the coupling of a $[422] 5 / 2^{+}$ proton to an axially symmetric core. The results suggest that the deformation is increasing slightly from $\epsilon_{2}=0.41$ for ${ }^{99} \mathrm{Y}$ to $\epsilon_{2}=0.43$ for ${ }^{101} \mathrm{Y}$. This observation is consistent with the small decrease of the energy of the $2_{1}^{+}$state from $144 \mathrm{keV}$ in ${ }^{98} \mathrm{Sr}$ to $129 \mathrm{keV}$ in ${ }^{100} \mathrm{Sr}$ and an increase of the $B\left(E 2 ; 2_{1}^{+} \rightarrow 0_{1}^{+}\right)$value from $0.259(8) e^{2} \mathrm{~b}^{2}$ [10] to $0.284(16)$ $e^{2} \mathrm{~b}^{2}$ [64]. The good agreement of the calculations with the experimental values for ${ }^{99} \mathrm{Y}$ and ${ }^{101} \mathrm{Y}$ is achieved without 

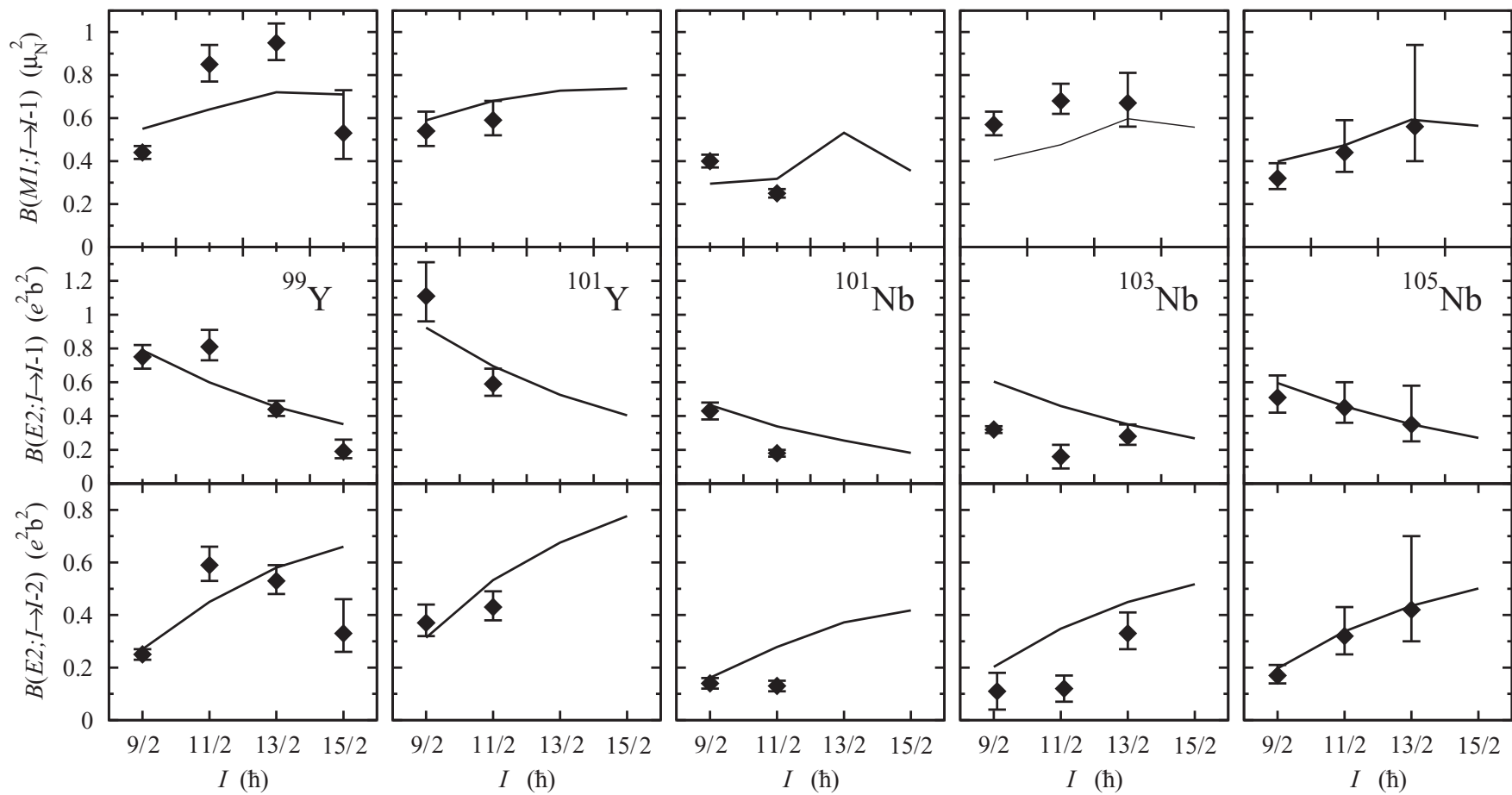

FIG. 16. Reduced transition probabilities for the $\Delta I=1$ and $\Delta I=2$ transitions in the ground-state bands of ${ }^{99} \mathrm{Y},{ }^{101} \mathrm{Y},{ }^{101} \mathrm{Nb},{ }^{103} \mathrm{Nb}$, and ${ }^{105} \mathrm{Nb}$. The experimental values are compared to results from triaxial particle-rotor calculations (solid lines).

scaling the Coriolis interaction or the intrinsic $g_{s}$ factor. Earlier particle-rotor calculations were also able to reproduce branching ratios in the ground-state band of ${ }^{99} \mathrm{Y}$ without renormalizing the $g_{s}$ factor [65].

The $\mathrm{Nb}$ isotopes exhibit a stronger signature splitting than the $Y$ isotopes and cannot be described without triaxial deformation of the core. The fit of the energies was improved by introducing a Coriolis attenuation of $\xi \approx 0.8$. The deformation parameter $\epsilon_{2}$ is found to be similar for all $\mathrm{Nb}$ isotopes under study and somewhat smaller compared to the $\mathrm{Y}$ isotopes. The results are consistent with the observed trend of decreasing deformation and increasing triaxility with increasing proton number from $\mathrm{Y}(Z=39)$ to $\mathrm{Rh}(Z=45)$ [18]. The results furthermore suggest that the $\gamma$ deformation of the $\mathrm{Nb}$ isotopes decreases with increasing neutron number.

The onset of triaxial deformation at $Z \approx 42$ is well established. Rotational bands with $K=2$ based on low-lying $2_{2}^{+}$ states are known in ${ }^{104} \mathrm{Mo},{ }^{106} \mathrm{Mo}$, and ${ }^{108} \mathrm{Mo}$ [66]. Applying

TABLE IV. Parameters used for the triaxial particle-rotor calculations: deformation parameters $\epsilon_{2}$ and $\gamma$, and scaling parameters for the moment of inertia, Coriolis attenuation, and intrinsic $g_{s}$ factor.

\begin{tabular}{lcrlll}
\hline \hline & $\epsilon_{2}$ & $\gamma$ & $E\left(2^{+}\right)$ & $\xi$ & $n_{g s}$ \\
\hline${ }^{99} \mathrm{Y}$ & 0.41 & $0^{\circ}$ & 0.144 & 1.0 & 1.0 \\
${ }^{101} \mathrm{Y}$ & 0.43 & $0^{\circ}$ & 0.15 & 1.0 & 1.0 \\
${ }^{101} \mathrm{Nb}$ & 0.35 & $19^{\circ}$ & 0.20 & 0.85 & 0.95 \\
${ }^{103} \mathrm{Nb}$ & 0.37 & $15^{\circ}$ & 0.16 & 0.8 & 0.95 \\
${ }^{105} \mathrm{Nb}$ & 0.36 & $13^{\circ}$ & 0.16 & 0.8 & 0.95 \\
${ }^{107} \mathrm{Nb}$ & 0.37 & $9^{\circ}$ & 0.152 & 0.82 & 0.95 \\
\hline \hline
\end{tabular}

the rigid triaxial rotor model, one obtains triaxiality parameters of $\gamma=15^{\circ}-20^{\circ}$ from the energies and branching ratios of these bands, which is similar to the values obtained from the particle-rotor model for the odd-mass $\mathrm{Nb}$ isotopes. There is, furthermore, evidence of two-phonon $\gamma$-vibrational bands in ${ }^{104}$ Mo [67], ${ }^{106}$ Mo [68], and ${ }^{108}$ Mo [69]. Similar bands were reported in ${ }^{103} \mathrm{Nb}$ [37] and ${ }^{105} \mathrm{Nb}$ [38] and interpreted as first examples for two-phonon $\gamma$-vibrational bands in odd-mass nuclei. Because of the similarity to the Mo isotopes and the fact that the $\mathrm{Zr}$ nuclei are well described as axially symmetric rotors, it may be more appropriate to think of the odd-mass $\mathrm{Nb}$ nuclei as proton holes coupled to a triaxially deformed $\mathrm{Mo}$ core instead of a proton particle coupled to a $\mathrm{Zr}$ core.

Although there is good agreement between the transition probabilities calculated with the triaxial particle-rotor model using the parameters given in Table IV and the experimental lifetimes and branching ratios, there is some indication that the deformation used in the particle-rotor calculations may be somewhat too large. The calculated spectroscopic quadrupole moments for the ground states are systematically larger than the experimental values obtained by laser spectroscopy [32,39]. The calculated spectroscopic quadrupole moments for ${ }^{99} \mathrm{Y}$ and ${ }^{101} \mathrm{Y}$ are $Q_{s}=+1.83 \mathrm{eb}$ and $Q_{s}=+1.98 \mathrm{eb}$, whereas the experimental values are $Q_{s}=+1.55(17) e \mathrm{~b}$ and $Q_{s}=+1.53(17) e b[32]$, respectively. For ${ }^{101} \mathrm{Nb}$ and ${ }^{103} \mathrm{Nb}$ the calculations yield $Q_{s}=+1.40 \mathrm{eb}$ and $Q_{s}=+1.61 \mathrm{eb}$, compared to the experimental values $Q_{s}=+1.05(7)$ and $Q_{s}=+1.08$ (9) [39], respectively. The quadrupole deformations used in the particle-rotor calculations for ${ }^{99} \mathrm{Y}$ and ${ }^{101} \mathrm{Y}$ are also larger by a similar amount compared to the intrinsic deformation of the neighboring even-even nuclei that can be extracted from the transition probabilities in their ground-state 
bands using the rotational model [11]. Calculations by Möller et al. based on the finite-range liquid drop model with shell corrections using a folded Yukawa potential find ground-state deformations of $\epsilon_{2}=0.33$ for ${ }^{99} \mathrm{Y}$ and $\epsilon_{2}=0.34$ for ${ }^{101} \mathrm{Y}$ [70], again somewhat smaller than the results found in this work. For the $\mathrm{Nb}$ iosotopes under study, Möller et al. find ground-state deformations ranging from $\epsilon_{2}=0.34$ to 0.36 , more consistent with the present results.

The magnetic moments for the ground states of both the $\mathrm{Y}$ and $\mathrm{Nb}$ isotopes from the particle-rotor calculation are also approximately $15 \%$ larger than the values measured by laser spectroscopy [32,39]. It was not possible to find parameters of the particle-rotor model that describe all experimental values simultaneously. If the $g_{s}$ factor is reduced to fit the experimental magnetic moment of the ground state, the calculated lifetimes are significantly longer than the experimental values. If, on the other hand, the deformation parameters are reduced to fit the experimental spectroscopic quadrupole moment of the ground state, the excitation energies within the band are not very well reproduced. These discrepancies seem to indicate the limitations of a rigid particle-rotor model, that does not include any degrees of freedom of the core. Another limitation may be related to shape coexistence, which is known to be important in this mass region, but cannot be treated in the model. Nevertheless, the overall agreement between the model and the experimental results is remarkable, and the $\mathrm{Y}$ and $\mathrm{Nb}$ isotopes with $N \geqslant 60$ may serve as textbook examples of particle-rotor coupling.

\section{SUMMARY AND CONCLUSIONS}

Lifetimes in the picosecond range were measured for the ground-state rotational bands in ${ }^{99} \mathrm{Y},{ }^{101} \mathrm{Y},{ }^{101} \mathrm{Nb},{ }^{103} \mathrm{Nb}$, and ${ }^{105} \mathrm{Nb}$ in an experiment at GANIL. The recoil-distance Doppler shift technique was for the first time combined with fusion-fission and transfer-fission reactions in inverse kinematics, populating a very wide range of neutron-rich nuclides in the same experiment. The fission fragments were identified event by event in mass number $A$ and atomic number $Z$ using the VAMOS ++ spectrometer after passing through a degrader foil that was placed at variable distances from the target. $\gamma$ rays emitted at the target position were measured with the EXOGAM array of segmented Ge Clover detectors and correlated with the uniquely identified fission fragments.

Combining the lifetimes of the excited states with the $\gamma$-ray branching ratios, it was possible to determine absolute $B(M 1)$ and $B(E 2)$ values for transitions in the strongly coupled bands built on the ground state of the odd-mass $\mathrm{Y}$ and $\mathrm{Nb}$ isotopes. Using the rotational model, it was furthermore possible to extract the intrinsic quadrupole moments $Q_{0}$ and gyromagnetic factors $g_{K}-g_{R}$ for individual states within the bands. It was found that both the quadrupole moments and the $g$ factors are constant within the bands, indicating that the rotational model is a valid approximation. The extracted $g$ factors support the previously assigned [422]5/2+ Nilsson configuration for the ground-state bands in all odd-mass $\mathrm{Y}$ and $\mathrm{Nb}$ isotopes under study. It was furthermore possible to correlate $\gamma$-ray transitions with ${ }^{107} \mathrm{Nb}$ and identify excited states in this nucleus for the first time.

Triaxial particle-rotor calculations were carried out in order to interpret the experimental results on the basis of the underlying nuclear shape. In the calculations the quadrupole deformation parameters $\epsilon_{2}$ and $\gamma$ were fitted to reproduce the energies of the states in the ground-state bands and their $\gamma$-ray branching ratios and lifetimes. The results of the calculations again confirmed the $[422] 5 / 2^{+}$configuration for the ground-state bands of all nuclides under study. It was found that the bands in the $\mathrm{Y}$ isotopes are best reproduced by a prolate core with axial symmetry $\left(\gamma=0^{\circ}\right)$ and a relatively large deformation of $\epsilon_{2} \approx 0.4$. For the $\mathrm{Nb}$ isotopes a moderate $\gamma$ deformation was required to reproduce the experimental data, and the quadrupole deformation $\epsilon_{2}$ was found to be slightly smaller compared to the $\mathrm{Y}$ isotopes. The results are consistent with the large deformations found in the neighboring eveneven isotopes with $N \geqslant 60$ and the appearance of triaxiality in the Mo isotopes. The fitting of deformation parameters in the particle-rotor calculations suggests that the $\gamma$ deformation for the $\mathrm{Nb}$ isotopes is decreasing with increasing neutron number.

The results demonstrate the power of combining RDDS lifetime measurements with fission reactions in inverse kinematics and the identification of fission fragments in a magnetic spectrometer, which allows measuring picosecond lifetimes in a wide range of neutron-rich nuclei in one single experiment. The experiment yielded many more new lifetime results in both even-even and odd-even nuclei, which will be published separately. The experiment was limited by the relatively low $\gamma$-ray efficiency at Doppler-sensitive backward angles. The $\gamma$ efficiency can be significantly improved by coupling the AGATA [71] $\gamma$-ray tracking detector array with VAMOS ++ . With higher efficiency at backward angles it will be possible to analyze $\gamma-\gamma$ coincidences, eliminating the influence of unknown feeding, and extend the measurements to even more neutron-rich isotopes.

\section{ACKNOWLEDGMENTS}

We would like to thank the technical teams at GANIL for their support during the experiment and the authors of Ref. [20] for optimizing the setup of VAMOS ++ and EXOGAM. We thank A. Navin for help and useful discussions. This work has been supported by the European Community FP7 "Capacities" Integrated Infrastructure Initiative, contract ENSAR No. 262010, by the Research Council of Norway under project grant 213442, and by Deutsche Forschungsgemeinschaft (DFG) project DE 1516/3-1.
[1] B. Pritychenko, M. Birch, B. Singh, and M. Horoi, At. Data Nucl. Data Tables 107, 1 (2016).
[2] I. Angeli and K. Marinova, At. Data Nucl. Data Tables 99, 69 (2013). 
[3] S. Naimi, G. Audi, D. Beck, K. Blaum, C. Böhm, C. Borgmann, M. Breitenfeldt, S. George, F. Herfurth, A. Herlert et al., Phys. Rev. Lett. 105, 032502 (2010).

[4] M. Albers, N. Warr, K. Nomura, A. Blazhev, J. Jolie, D. Mücher, B. Bastin, C. Bauer, C. Bernards, L. Bettermann et al., Phys. Rev. Lett. 108, 062701 (2012).

[5] R. Sheline, I. Ragnarsson, and S. Nilsson, Phys. Lett. B 41, 115 (1972).

[6] T. Kibédi and R. Spear, At. Data Nucl. Data Tables 89, 77 (2005).

[7] J. Wood, E. Zganjar, C. D. Coster, and K. Heyde, Nucl. Phys. A 651, 323 (1999).

[8] F. Schussler, J. Pinston, E. Monnand, A. Moussa, G. Jung, E. Koglin, B. Pfeiffer, R. Janssens, and J. van Klinken, Nucl. Phys. A 339, 415 (1980).

[9] G. Lhersonneau, B. Pfeiffer, K.-L. Kratz, T. Enqvist, P. P. Jauho, A. Jokinen, J. Kantele, M. Leino, J. M. Parmonen, H. Penttilä, and J. Äystö (ISOLDE Collaboration), Phys. Rev. C 49, 1379 (1994).

[10] E. Clément, M. Zielińska, A. Görgen, W. Korten, S. Péru, J. Libert, H. Goutte, S. Hilaire, B. Bastin, C. Bauer et al., Phys. Rev. Lett. 116, 022701 (2016).

[11] E. Clément, M. Zielińska, S. Péru, H. Goutte, S. Hilaire, A. Görgen, W. Korten, D. Doherty, B. Bastin, C. Bauer et al., Phys. Rev. C 94, 054326 (2016).

[12] J. Hamilton, A. Ramayya, S. Zhu, G. Ter-Akopian, Y. Oganessian, J. Cole, J. Rasmussen, and M. Stoyer, Prog. Part. Nucl. Phys. 35, 635 (1995).

[13] A. Kumar and M. R. Gunye, Phys. Rev. C 32, 2116 (1985).

[14] R. Meyer, E. Monnand, J. Pinston, F. Schussler, I. Ragnarsson, B. Pfeiffer, H. Lawin, G. Lhersonneau, T. Seo, and K. Sistemich, Nucl. Phys. A 439, 510 (1985).

[15] I. Ragnarsson, Acta Phys. Pol. B 27, 33 (1996).

[16] E. S. Paul, P. J. Twin, A. O. Evans, A. Pipidis, M. A. Riley, J. Simpson, D. E. Appelbe, D. B. Campbell, P. T. W. Choy, R. M. Clark et al., Phys. Rev. Lett. 98, 012501 (2007).

[17] J. Shannon, W. Phillips, J. Durell, B. Varley, W. Urban, C. Pearson, I. Ahmad, C. Lister, L. Morss, K. Nash et al., Phys. Lett. B 336, 136 (1994).

[18] S. Zhu, Y. Luo, J. Hamilton, J. Rasmussen, A. Ramayya, J. Hwang, H. Ding, X. Che, Z. Jiang, P. Gore et al., Prog. Part. Nucl. Phys. 59, 329 (2007).

[19] J. Jolie, J.-M. Régis, D. Wilmsen, S. Ahmed, M. Pfeiffer, N. Saed-Samii, N. Warr, A. Blanc, M. Jentschel, U. Köster et al., EPJ Web Conf. 93, 01014 (2015).

[20] A. Navin, M. Rejmund, C. Schmitt, S. Bhattacharyya, G. Lhersonneau, P. V. Isacker, M. Caamano, E. Clément, O. Delaune, F. Farget, G. de France, and B. Jacquot, Phys. Lett. B 728, 136 (2014).

[21] A. Navin and M. Rejmund, Yearbook of Science and Technology 2014 (McGraw-Hill Education, New York, 2013), pp. 137-141.

[22] A. Görgen, J. Phys. G: Nucl. Part. Phys. 37, 103101 (2010).

[23] A. Görgen and W. Korten, J. Phys. G: Nucl. Part. Phys. 43, 024002 (2016).

[24] C. Sotty, M. Zielińska, G. Georgiev, D. L. Balabanski, A. E. Stuchbery, A. Blazhev, N. Bree, R. Chevrier, S. Das Gupta, J. M. Daugas et al., Phys. Rev. Lett. 115, 172501 (2015).

[25] H. Mach, R. Gill, and M. Moszyński, Nucl. Instrum. Methods Phys. Res., Sect. A 280, 49 (1989).

[26] M. Moszyński and H. Mach, Nucl. Instrum. Methods Phys. Res., Sect. A 277, 407 (1989).
[27] A. G. Smith, W. R. Phillips, J. L. Durell, W. Urban, B. J. Varley, C. J. Pearson, J. A. Shannon, I. Ahmad, C. J. Lister, L. R. Morss et al., Phys. Rev. Lett. 73, 2540 (1994).

[28] A. G. Smith, R. M. Wall, D. Patel, G. S. Simpson, D. M. Cullen, J. L. Durell, S. J. Freeman, J. C. Lisle, J. F. Smith, B. J. Varley et al., J. Phys. G: Nucl. Part. Phys. 28, 2307 (2002).

[29] E. Monnand, J. A. Pinston, F. Schussler, B. Pfeiffer, H. Lawin, G. Battistuzzi, K. Shizuma, and K. Sistemich, Z. Phys. A 306, 183 (1982).

[30] F. K. Wohn, J. C. Hill, R. F. Petry, H. Dejbakhsh, Z. Berant, and R. L. Gill, Phys. Rev. Lett. 51, 873 (1983).

[31] Y. X. Luo, J. O. Rasmussen, I. Stefanescu, A. Gelberg, J. H. Hamilton, A. V. Ramayya, J. K. Hwang, S. J. Zhu, P. M. Gore, D. Fong et al., J. Phys. G: Nucl. Part. Phys. 31, 1303 (2005).

[32] B. Cheal, M. Gardner, M. Avgoulea, J. Billowes, M. Bissell, P. Campbell, T. Eronen, K. Flanagan, D. Forest, J. Huikari et al., Phys. Lett. B 645, 133 (2007).

[33] H. Mach, F. K. Wohn, M. Moszyński, R. L. Gill, and R. F. Casten, Phys. Rev. C 41, 1141 (1990).

[34] T. Seo, A. M. Schmitt, H. Ahrens, J. P. Bocquet, N. Kaffrell, H. Lawin, G. Lhersonneau, R. A. Meyer, K. Shizuma, K. Sistemich et al., Z. Phys. A 315, 251 (1984).

[35] T. Seo, R. A. Meyer, and K. Sistemich, in Nuclear Structure of the Zirconium Region: Proceedings of the International Workshop, Bad Honnef, Federal Republic of Germany, edited by J. Eberth, R. A. Meyer, and K. Sistemich (Springer, Berlin, 1988), pp. 349-354.

[36] M. Hotchkis, J. Durell, J. Fitzgerald, A. Mowbray, W. Phillips, I. Ahmad, M. Carpenter, R. Janssens, T. Khoo, E. Moore et al., Nucl. Phys. A 530, 111 (1991).

[37] J.-G. Wang, S.-J. Zhu, J. Hamilton, A. Ramayya, J. Hwang, S. Liu, K. Li, Y. Luo, J. Rasmussen, I. Lee et al., Phys. Lett. B 675, 420 (2009).

[38] H. J. Li, S. J. Zhu, J. H. Hamilton, A. V. Ramayya, J. K. Hwang, Y. X. Liu, Y. Sun, Z. G. Xiao, E. H. Wang, J. M. Eldridge et al., Phys. Rev. C 88, 054311 (2013).

[39] B. Cheal, K. Baczynska, J. Billowes, P. Campbell, F. C. Charlwood, T. Eronen, D. H. Forest, A. Jokinen, T. Kessler, I. D. Moore et al., Phys. Rev. Lett. 102, 222501 (2009).

[40] H. Ohm, M. Liang, U. Paffrath, B. De Sutter, K. Sistemich, A. M. Schmitt, N. Kaffrell, N. Trautmann, T. Seo, K. Shizuma et al., Z. Phys. A 340, 5 (1991).

[41] M. Liang, H. Ohm, B. De Sutter, and K. Sistemich, Z. Phys. A 344, 357 (1993).

[42] A. G. Smith, J. L. Durell, W. R. Phillips, W. Urban, P. Sarriguren, and I. Ahmad, Phys. Rev. C 86, 014321 (2012).

[43] A. Dewald, S. Harissopulos, and P. von Brentano, Z. Phys. A 334, 163 (1989).

[44] A. Dewald, O. Möller, and P. Petkov, Prog. Part. Nucl. Phys. 67, 786 (2012).

[45] S. Pullanhiotan, M. Rejmund, A. Navin, W. Mittig, and S. Bhattacharyya, Nucl. Instrum. Methods Phys. Res., Sect. A 593, 343 (2008).

[46] J. Simpson, F. Azaiez, G. de France, J. Fouan, J. Gerl, R. Julin, W. Korten, P. Nolan, B. Nyakó, G. Sletten, P. Walker, and the EXOGAM Collaboration, Acta Phys. Hungarica 11, 159 (2000).

[47] M. Caamaño, O. Delaune, F. Farget, X. Derkx, K.-H. Schmidt, L. Audouin, C.-O. Bacri, G. Barreau, J. Benlliure, E. Casarejos et al., Phys. Rev. C 88, 024605 (2013).

[48] M. Rejmund, B. Lecornu, A. Navin, C. Schmitt, S. Damoy, O. Delaune, J. Enguerrand, G. Fremont, P. Gangnant, L. Gaudefroy 
et al., Nucl. Instrum. Methods Phys. Res., Sect. A 646, 184 (2011).

[49] S. Pullanhiotan, A. Chatterjee, B. Jacquot, A. Navin, and M. Rejmund, Nucl. Instrum. Methods Phys. Res., Sect. B 266, 4148 (2008).

[50] L. Northcliffe and R. Schilling, At. Data Nucl. Data Tables 7, 233 (1970).

[51] M. Hackstein and A. Dewald, Nucl. Instrum. Methods Phys. Res., Sect. A 738, 93 (2014).

[52] D. De Frenne, Nucl. Data Sheets 110, 2081 (2009).

[53] J. K. Hwang, A. V. Ramayya, J. Gilat, J. H. Hamilton, L. K. Peker, J. O. Rasmussen, J. Kormicki, T. N. Ginter, B. R. S. Babu, C. J. Beyer et al., Phys. Rev. C 58, 3252 (1998).

[54] J. Pereira, S. Hennrich, A. Aprahamian, O. Arndt, A. Becerril, T. Elliot, A. Estrade, D. Galaviz, R. Kessler, K.-L. Kratz et al., Phys. Rev. C 79, 035806 (2009).

[55] G. Lorusso, S. Nishimura, Z. Y. Xu, A. Jungclaus, Y. Shimizu, G. S. Simpson, P.-A. Söderström, H. Watanabe, F. Browne, P. Doornenbal et al., Phys. Rev. Lett. 114, 192501 (2015).

[56] J. G. Wang, S. J. Zhu, J. H. Hamilton, A. V. Ramayya, J. K. Hwang, K. Li, Y. X. Luo, J. O. Rasmussen, I. Y. Lee, H. B. Ding et al., Phys. Rev. C 78, 014313 (2008).

[57] Y. X. Luo, J. O. Rasmussen, J. H. Hamilton, A. V. Ramayya, E. Wang, Y. X. Liu, C. F. Jiao, W. Y. Liang, F. R. Xu, Y. Sun et al., Phys. Rev. C 89, 044326 (2014).

[58] D. J. Rowe, Nuclear Collective Motion: Models and Theory (Methuen, London, 1970).

[59] I. Ragnarsson and P. B. Semmes, Hyperfine Interact. 43, 423 (1988).
[60] T. Bengtsson and I. Ragnarsson, Nucl. Phys. A 436, 14 (1985).

[61] S. Larsson, G. Leander, and I. Ragnarsson, Nucl. Phys. A 307, 189 (1978).

[62] I. Ragnarsson and R. K. Sheline, Phys. Scr. 29, 385 (1984).

[63] P. Semmes, in Proceedings of the International Workshop on Nuclear Structure Models, edited by R. Bengtsson, J. Draayer, and W. Nazarewicz (World Scientific, Singapore, 1992), p. 287.

[64] G. Lhersonneau, H. Gabelmann, N. Kaffrell, K.-L. Kratz, B. Pfeiffer, and K. Heyde, Z. Phys. A 337, 143 (1990).

[65] F. K. Wohn, J. C. Hill, and R. F. Petry, Phys. Rev. C 31, 634 (1985).

[66] H. Hua, C. Y. Wu, D. Cline, A. B. Hayes, R. Teng, R. M. Clark, P. Fallon, A. Goergen, A. O. Macchiavelli, and K. Vetter, Phys. Rev. C 69, 014317 (2004).

[67] A. Guessous, N. Schulz, M. Bentaleb, E. Lubkiewicz, J. L. Durell, C. J. Pearson, W. R. Phillips, J. A. Shannon, W. Urban, B. J. Varley et al., Phys. Rev. C 53, 1191 (1996).

[68] A. Guessous, N. Schulz, W. R. Phillips, I. Ahmad, M. Bentaleb, J. L. Durell, M. A. Jones, M. Leddy, E. Lubkiewicz, L. R. Morss et al., Phys. Rev. Lett. 75, 2280 (1995).

[69] H.-B. Ding, S.-J. Zhu, J. H. Hamilton, A. V. Ramayya, J. K. Hwang, Y. X. Luo, J. O. Rasmussen, I. Y. Lee, X.-L. Che, J.-G. Wang, and Q. Xu, Chin. Phys. Lett. 24, 1517 (2007).

[70] P. Möller, A. Sierk, T. Ichikawa, and H. Sagawa, At. Data Nucl. Data Tables 109-110, 1 (2016).

[71] S. Akkoyun, A. Algora, B. Alikhani, F. Ameil, G. de Angelis, L. Arnold, A. Astier, A. Ataç, Y. Aubert, C. Aufranc et al. (AGATA Collaboration), Nucl. Instrum. Methods Phys. Res., Sect. A 668, 26 (2012). 